\title{
Research Article Distortion-Based Link Adaptation for Wireless Video Transmission
}

\author{
Pierre Ferré, ${ }^{1}$ James Chung-How, ${ }^{2}$ David Bull, ${ }^{1}$ and Andrew Nix ${ }^{1}$ \\ ${ }^{1}$ Centre for Communications Research, University of Bristol, Woodland Road, Bristol BS8 1UB, UK \\ ${ }^{2}$ ProVision Communication Technologies Limited, 3 Chapel Way, St. Anne's, Bristol BS4 4EU, UK
}

Correspondence should be addressed to Pierre Ferré, pierre.ferre@bristol.ac.uk

Received 15 October 2007; Accepted 10 March 2008

Recommended by F. Babich

\begin{abstract}
Wireless local area networks (WLANs) such as IEEE 802.11a/g utilise numerous transmission modes, each providing different throughputs and reliability levels. Most link adaptation algorithms proposed in the literature (i) maximise the error-free data throughput, (ii) do not take into account the content of the data stream, and (iii) rely strongly on the use of ARQ. Low-latency applications, such as real-time video transmission, do not permit large numbers of retransmission. In this paper, a novel link adaptation scheme is presented that improves the quality of service (QoS) for video transmission. Rather than maximising the error-free throughput, our scheme minimises the video distortion of the received sequence. With the use of simple and local rate distortion measures and end-to-end distortion models at the video encoder, the proposed scheme estimates the received video distortion at the current transmission rate, as well as on the adjacent lower and higher rates. This allows the system to select the link-speed which offers the lowest distortion and to adapt to the channel conditions. Simulation results are presented using the MPEG-4/AVC H.264 video compression standard over IEEE 802.11g. The results show that the proposed system closely follows the optimum theoretic solution.
\end{abstract}

Copyright (C) 2008 Pierre Ferré et al. This is an open access article distributed under the Creative Commons Attribution License, which permits unrestricted use, distribution, and reproduction in any medium, provided the original work is properly cited.

\section{INTRODUCTION}

Low-latency video transmission is highly demanding in terms of the performance of all layers in the protocol stack. Over the last decade, research has mainly focused on enhancements to each individual layer without considering cross-layer interactions. Adapting the source coding according to the channel and network conditions (and vice versa) [1] via the cross-layer exchange of information has only recently been investigated. In [2, 3], van der Schaar et al. develop a cross-layer optimisation that combines application layer forward error correction (FEC), adaptive medium access control (MAC) retransmission and adaptive packetisation for video transmission over an IEEE $802.11 \mathrm{~b}$ network. In [4], the authors discuss the challenges and principles of cross-layer optimised multimedia transmission. The choice of optimal modulation using Application/MAC/PHY interactions for video over IEEE 802.11b [5] is discussed as well as the choice of modulation scheme for optimal power consumption. Moreover, the authors stress the fact that an optimal solution for throughput may not be appropriate for multimedia transmission. In [6], Setton et al. detail the basis of a cross-layer framework where packet size is dynamically adapted for a given link layer and channel condition. For a given packet length, the proposed scheme optimises the link layer parameters, such as the constellation and the symbol rate, in order to optimise the throughput. In $[7,8]$, the authors develop a hybrid link adaptation mechanism, combining different link adaptation techniques and using a cross-layering signalling system aimed at improving the received video quality. In [9], a cross-layer architecture is developed for MPEG-4/AVC H.264 [10] video over the IEEE 802.11e [11] MAC layer by assigning priority values to network abstraction layer (NAL) units that are then converted into priority accesses, specific to the MAC layer. However, with the exception of $[3,4,7]$, adaptive link and MAC layer techniques, involving coding rate and modulation adaptation, are rarely considered in the design of cross-layer systems.

This paper investigates a link adaptation mechanism appropriate for the delivery of low-latency real-time video without relying on retransmission. Distortion models are 


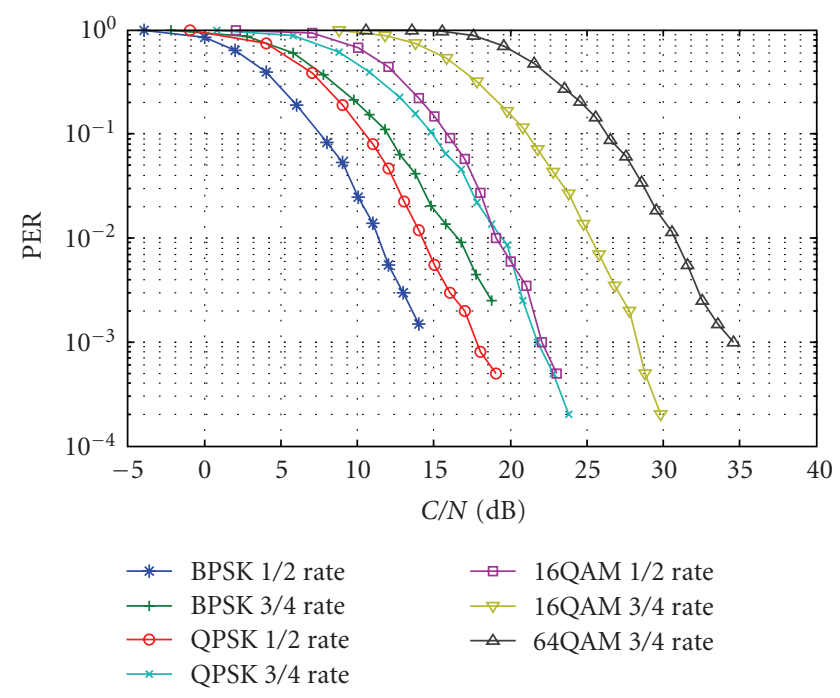

FIgURE 1: IEEE $802.11 \mathrm{a} / \mathrm{g}$ PER performance, ETSI, BRAN Channel A [14], 825 byte packets.

developed and simulations are performed in order to evaluate the proposed scheme. The algorithm presented uses cross-layer exchange of information and is designed to optimise perceptual video quality (by minimising the perceived distortion) at the receiver. The paper is organised as follows. Section 2 presents the principles of link adaptation in IEEE 802.11 WLANs and describes the existing algorithms. The models used for the estimation of the distortion are described and validated in Section 3. Section 4 details the proposed link adaptation algorithms, and results are presented in Section 5. Finally, Section 6 concludes the paper.

\section{LINK ADAPTATION IN IEEE 802.11 WLANs}

\subsection{IEEE 802.11a/g PHY and MAC}

The PHY layers of COFDM-based WLANs at $2.4 \mathrm{GHz}$ and $5 \mathrm{GHz}$, such as IEEE 802.11g [12] and IEEE 802.11a [13], respectively, offer numerous coding rates and modulation schemes, each providing different throughputs and reliability levels. Table 1 summarises the different link-speeds (commonly called operating modes) available for the IEEE 802.11a/g PHY layers. These range from BPSK $1 / 2$ rate (mode 1) which provides a nominal bit rate of $6 \mathrm{Mbps}$, to $64 \mathrm{QAM} 3 / 4$ rate (mode 7 ), with a nominal bit rate of $54 \mathrm{Mbps}$. The BPSK $1 / 2$ rate mode provides a more reliable transmission link than the 64 QAM 3/4 rate mode for a given received power level. Figure 1 shows the packet error rate (PER) performance versus power level (carrierto-noise ratio $(\mathrm{C} / \mathrm{N})$ ) for the 7 link-speeds available in IEEE $802.11 \mathrm{a} / \mathrm{g}$ with a PHY packet length of 825 bytes (selected as a compromise between PHY PER performance and MAC layer throughput). Since the PER performance varies considerably between modes, the choice of operating link-speed is crucial to system performance. It should be noted that operating modes and link-speeds are equivalent and, in the remainder of this paper, both terms are used interchangeably.
Due to the range of operating modes available at the PHY layer, the ability for a system to adapt to the fluctuations of the environment (mobility, interference, and congestion) is vital to optimise overall performance. This ability to change link-speeds is used to control the reliability of the system and provides the radio with the ability to switch to a better configuration to improve the QoS of the transmission. Many parameters can be varied at the MAC and PHY level; examples include the maximum number of MAC level retries (or automatic repeat requests (ARQ)), the packet size, the operating mode (modulation, coding rate, link-speed), and the type and number of antennas. Neither the IEEE 802.11 MAC [15] nor the IEEE $802.11 \mathrm{a} / \mathrm{g}$ standards specifies an algorithm for dynamic rate switching. The IEEE 802.11 MAC only defines rules for the mode selection of the management frames and declares dynamic rate selection for user data beyond the scope of the specifications $[8,15,16]$. It is therefore left to manufacturers to implement their own switching algorithms and metrics, examples of these include throughput, PER or delay.

\subsection{Existing link adaptation algorithms and related work}

A simple link adaptation algorithm can be based on statistics about the transmitted data. Such schemes are known as Statistics-based automatic rate control algorithms $[7,8,16]$. These aim to provide the highest throughput $[17,18]$ since the statistics are directly related to user-level throughput. Other techniques use direct measurement of the link conditions, based for example on power levels which are closely related to the PER, and therefore to the throughput $[7,8]$.

\subsubsection{Statistics-based control}

(i) Throughput-based control: in these algorithms, a constant (small) fraction of data (up to 10\%) is sent at two adjacent link-speeds (lower and higher than the current rate). At the end of a decision window, the transmitter computes the different throughputs and a switch is made to the rate that provides the highest throughput. In order to have meaningful statistics, the decision window must be sufficiently long (approximately one second $[7,8]$ ).

(ii) PER-based control: in these algorithms, the PER of the transmitted data is used to select the link-speed. The PER can be determined by counting the ACKs of the IEEE 802.11 MAC frame received at the transmitter during a sliding decision window (a missing ACK means that the corresponding packet has not been received correctly). This approach was not designed for video transmission, and optimises the PER to achieve an improved throughput. It does not take into account the nature of the content and its timebounded requirements.

(iii) Retry-based control: in these algorithms, the decision metric used is the number of failed ARQs. If a transmission is unsuccessful after a certain number of 
TABLE 1: Mode-dependent parameters for IEEE 802.11a/g.

\begin{tabular}{ccccc}
\hline Operating mode & Modulation & Coding rate & Link-Speed in Mbps & Bit rate ratio with mode 1 \\
\hline 1 & BPSK & $1 / 2$ & 6 & 1 \\
2 & BPSK & $3 / 4$ & 9 & $3 / 2$ \\
3 & QPSK & $1 / 2$ & 12 & 2 \\
4 & QPSK & $3 / 4$ & 18 & 3 \\
5 & 16 QAM & $1 / 2$ & 24 & 4 \\
6 & 16 QAM & $3 / 4$ & 36 & 6 \\
7 & 64 QAM & $3 / 4$ & 54 & 9 \\
\hline
\end{tabular}

retries, $N_{\text {fail }}$, the link-speed is downscaled. Similarly, upscaling would occur after a certain number of successful contiguous transmissions, $N_{\text {success }}$ [19]. This method offers a very short response time to channel changes. Upscaling can also be implemented with a PER-based control scheme using a decision window. This has been developed under the name of AutoRate Fall Back (ARF) [20, 21] and has been designed to optimise the application throughput [19].

\subsubsection{SNR-based control}

In this method, the carrier-to-noise ratio $(\mathrm{C} / \mathrm{N})$, also known as the signal-to-noise ratio ( $\mathrm{SNR}$ ), is used to determine the transmission rate. The value of $\mathrm{C} / \mathrm{N}$ is directly related to the PER. The throughput at the PHY layer can be expressed as a function of the PER and can be estimated as in [22-24]:

$$
\text { Throughput }=R \times(1-\mathrm{PER}) \text {, }
$$

where $R$ is the operating link-speed (or nominal bit rate) (see Table 1). Link adaptation based on SNR/throughput is presented in Figure 2 for a MAC packet length of 825 bytes. The crossing points of the curves define the switching points (in terms of $\mathrm{C} / \mathrm{N}$ ) at which the system should up or downscale. A simple SNR-based algorithm would employ a look-up table (made available at the MAC) to obtain the best throughput for a given $\mathrm{C} / \mathrm{N}$ [25]. These tables could theoretically be generated off-line for different packet lengths for all modes, $\mathrm{C} / \mathrm{Ns}$ and different channel conditions. It should be noted that this assumes that ARQ is used for retransmitting packets until the packet is received correctly, or the maximum number of retries is reached (whichever comes first). Data are therefore received error-free but delays are incurred and the nature of the data is not taken into account.

\subsubsection{Other rate adaptation algorithms}

Several rate adaptation algorithms have been presented in the literature. A selection of these is presented here. A good review of link adaptation design guidelines can be found in [26], where the authors compare the merits of the more common algorithms to derive a mechanism overcoming their disadvantages. In [27], the authors develop

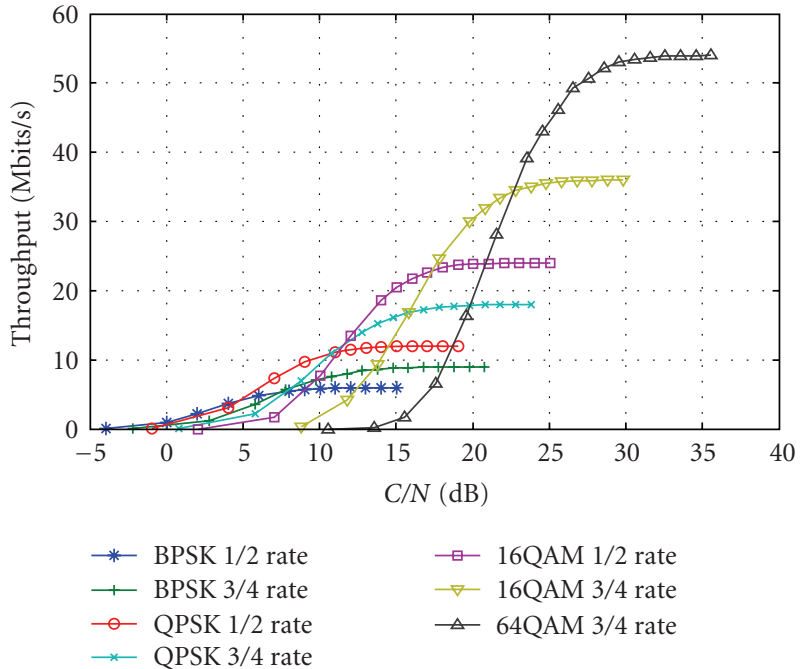

FIGURE 2: Link adaptation based on throughput, IEEE 802.11a/g, 825 byte packets.

the minimum energy transmission strategy (MiSer) scheme, which minimises the communication energy consumption by combining the transport power control with the PHY rate adaptation. In [28], the receiver-based autorate ( $\mathrm{R}$ $\mathrm{BAR}$ ) protocol is presented which optimises the application throughput [19], where the choice of transmission rate is made at the receiver based on its own stored statistics [21]. The information on the chosen rate is then transferred back to the transmitter via the CTS frame of the handshaking RTS/CTS. In $[29,30]$, the authors develop a hybrid automatic rate controller, combining a throughput-based rate controller with an SNR-based approach. By dynamically adjusting RSSI-look up tables, the algorithm selects the most appropriate rate. This scheme aims at improving throughput as well as reducing delay and PER, but is also able to adjust the transmitted video rate. A hardware solution is discussed in [7], together with video results. In [31], the authors derived an algorithm which allows differentiating packet loss due to channel errors from packet collisions. Using the RTS frame of IEEE 802.11 in an adaptive manner, the proposed system is more likely to make the correct rate adaptation. Variations of the above algorithms can be found in many papers, among which [25, 32-35] are notable. 
Almost all the reported link adaptation algorithms have been designed to provide throughput and/or PER performance improvements [18] and/or to reduce the power consumption. They do not take into account the nature of the transmitted data or the low-delay requirements common to real-time video applications. They strongly rely on the use of retransmission and do not consider transmission delays. Moreover, in the case of multimedia transmission, they also do not optimise the perceived video quality [4].

\subsection{Motivation}

In our previous work $[17,36]$, we have shown that existing algorithms are generally not suitable for low-latency video applications as (i) they do not take into account the nature of the transmitted data, and (ii) they are primarily designed to provide the highest throughput without regard for delay and retransmission. For video transmission where a strong reliance on ARQ is not desirable, a completely error-free communication is not essential when robust video compression techniques are applied. For example, it is possible to obtain an improved decoded video quality using a higher link-speed but with some degree of error, rather than an error-free video stream at a lower bitrate (using a lower link-speed). This is demonstrated in Figure 3 for the foreman sequence (average peak-to-peak signal-to-noise ratio (PSNR) over the whole sequence is shown here) for the case with no ARQ. Each mode can carry one video bit rate and, hence, higher modes support better video quality if the PER is sufficiently low. The overall quality of the received video sequence depends on a tradeoff between video bit-rate and error rate, as shown in Figure 4. For a given $\mathrm{C} / \mathrm{N}$ of $18 \mathrm{~dB}$, mode 1 provides error-free transmission at low video bit rates $(700 \mathrm{kbps}$ with a peak signal-to-noise ratio (PSNR) of $37.07 \mathrm{~dB}$ ), whereas mode 5 provides a transmission with a PER of $10^{-2}$ with a higher video bit rate $(4235 \mathrm{kbps})$. However, Figure 4(b) shows better resolution and presents a better PSNR (44.85 dB) than Figure 4(a) (37.07 dB). Impairments due to errors are insignificant and can not be noticed visually.

Whenever the MAC layer adapts its link-speed, the application layer also adapts its encoding rate, based on the following two assumptions:

(i) the ratios between the bit rates carried on each mode follow the ratios of the link-speeds available at the PHY layer for each mode, as shown in the last column of Table 1. In this way, similar PHY resources are used for each link-speed;

(ii) the maximum size of the video packet generated at the encoder is not modified. A nonadaptive packetsize assumption is the most realistic case for such a system.

Therefore, if mode 1 is used to stream video at $500 \mathrm{kbps}$, modes $2,3,4,5,6$, and 7 will carry video encoded at $750,1000,1500,2000,3000$, and $4500 \mathrm{kbps}$, respectively. As the $\mathrm{C} / \mathrm{N}$ increases, changing to higher link-speeds with a

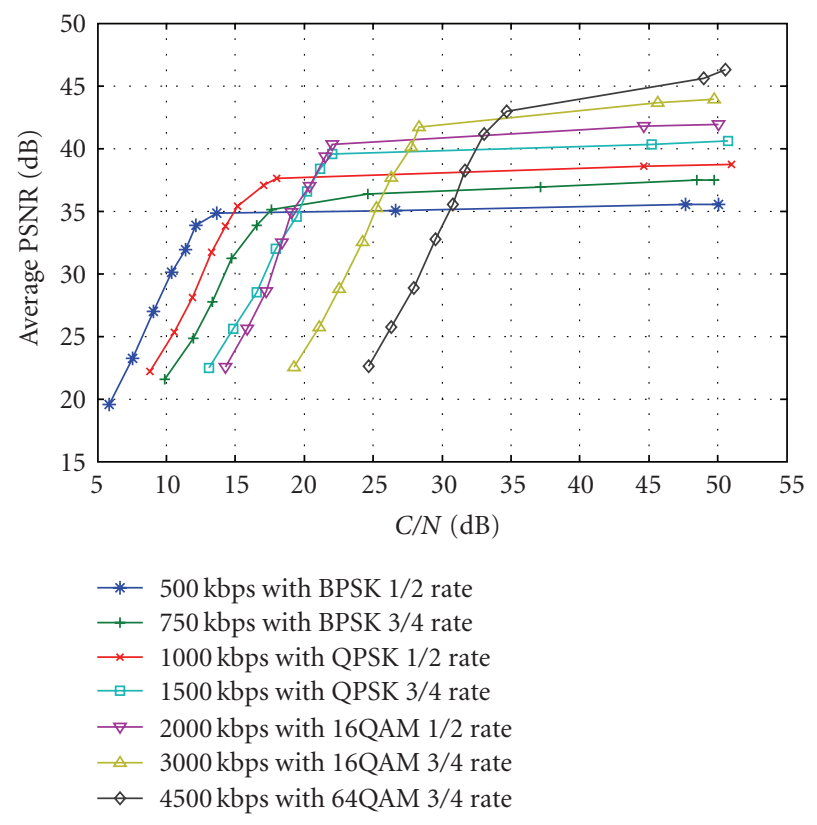

FIGURE 3: Video quality-based algorithm, foreman, NAL unit max size: 750 bytes.

higher bit rate provides a better PSNR. For example, the best-video quality is obtained with QPSK $1 / 2$ rate (mode 3) with $1000 \mathrm{kbps}$ at a $\mathrm{C} / \mathrm{N}$ of $17 \mathrm{~dB}$, with some degree of error, whereas BPSK $1 / 2$ rate with $500 \mathrm{kbps}$ is error-free. A natural and empirical switching point would therefore be based on PSNR; effectively selecting the link-speed with the highest PSNR at any time and for any $\mathrm{C} / \mathrm{N}$ level. However, in a realistic scenario, the decoder cannot derive PSNR because it does not have access to the original video reference. Moreover, PSNR performance depends on the content, the video bit rate, the concealment algorithm, and the packet length (amongst others).

A switching scheme using PER thresholds was presented by the authors in [17]. Comparisons of this approach with existing throughput-based solutions were made. The principle is shown in Figure 5 where it can be seen that switching occurs at lower PHY PERs for the video qualitybased algorithm. In [17], it was shown that parameters such as packet size, video rate, and content had a strong influence on the PER thresholds. A rigorous derivation of the PER thresholds was therefore found difficult to establish, and a practical design could not be proposed.

\subsection{Proposed approach}

Building on the preliminary work in [17], this paper investigates a rigorous switching scheme based on the received video distortion. The distortion measured here is to the mean square error (MSE) between the received and original pixels. This includes the encoding distortion (due to the coding, transform, and motion compensation operation of the encoder) as well as the end-to-end distortion (due to error propagation and error concealment). The 


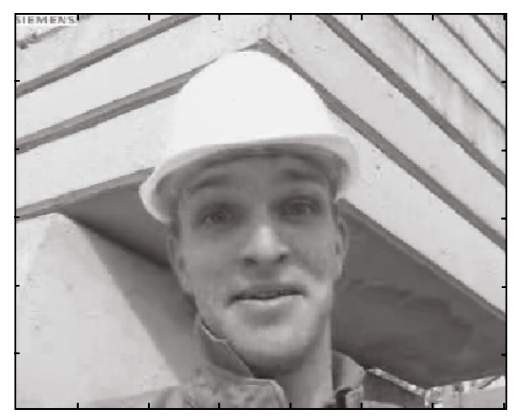

(a) Mode $1,700 \mathrm{~kb}, \mathrm{PER}=0, \mathrm{PSNR}=37.07 \mathrm{~dB}$

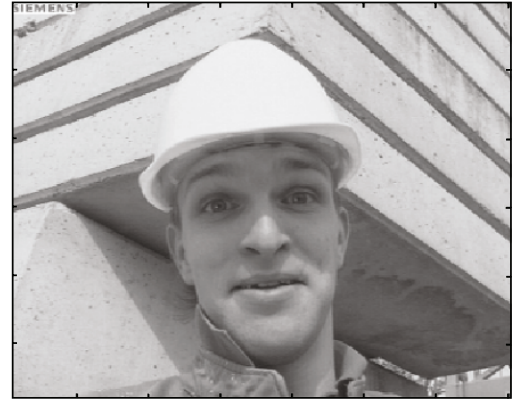

(b) Mode 5, $4235 \mathrm{kbps}, \mathrm{PER}=0.04, \mathrm{PSNR}=44.85 \mathrm{~dB}$

FIgUre 4: Foreman sequence, frame $30, \mathrm{C} / \mathrm{N}=18 \mathrm{~dB}$.

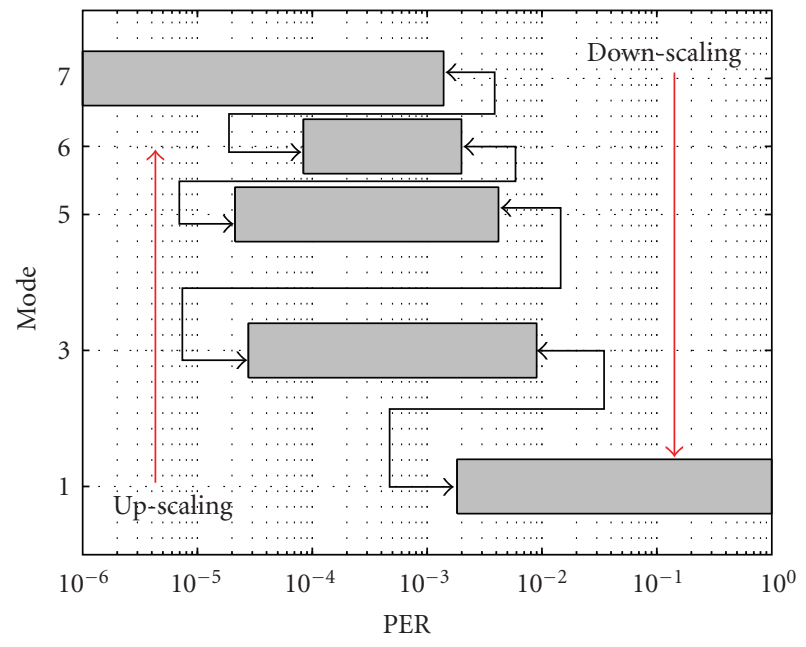

(a) Video quality-based

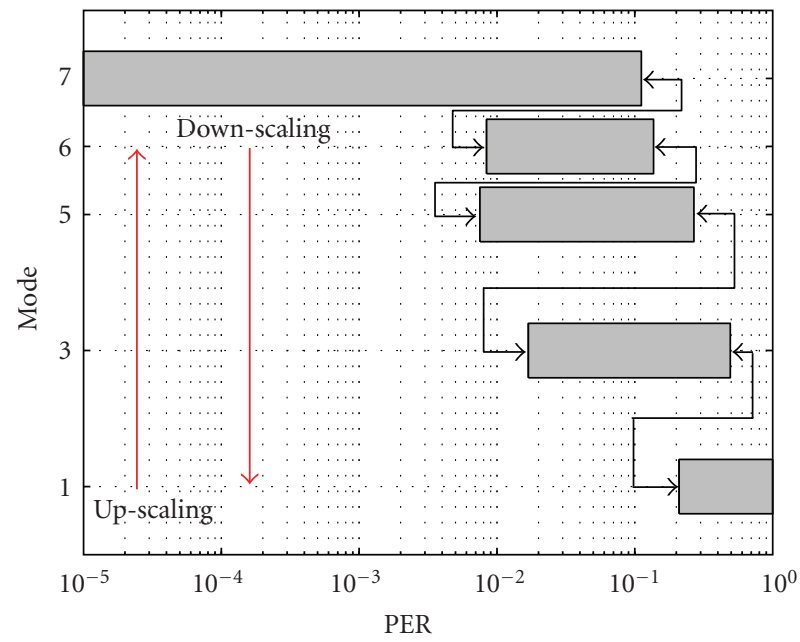

(b) Throughput-based

FIGURE 5: Switching points comparison, foreman.

same assumptions remain, that is, the ratio between the bit rates carried on each mode follows the ratio of the link-speeds available at the PHY layer for each mode; and the maximum size of the video packet generated at the encoder is not modified. Rather than using PSNR as a switching metric, the new scheme presented in this paper uses an estimate of the video distortion. The decision to switch from one link-speed to another is made upon the distortion experienced on the current mode, as well as the distortion on adjacent modes. For a given channel condition, the mode offering the lowest distortion, that is, the best video quality, is selected, as shown in Figure 6 (the average distortion over the whole sequence is shown here). Clearly, without a reference, the end-to-end distortions can not be computed at the transmitter and need to be estimated. A simple model to estimate the distortion at the current mode and at the two adjacent has been developed and is presented in the next section. The proposed approach operates on a group of pictures (GOP) basis, where distortions are estimated and switching decisions are made for each GOP.

\section{VIDEO TRANSMISSION MODEL DESCRIPTION}

To enable mode switching based on distortion we need to estimate (i) the distortion of the received sequence transmitted at the current rate, under the given channel conditions, and (ii) the distortions of the received sequence if transmitted at lower and higher rates, under their corresponding channel conditions. To do so, we need to model (i) the rate distortion curve of the sequence; and (ii) an endto-end distortion. The following discussion is based on the H.264 standard [10] which is used throughout the paper.

\subsection{Empirical rate distortion model}

Several accurate RD models have been presented in the literature [37-39]. However, these require trial encodings in order to determine sequence-dependent parameters (and hence cannot be used for practical systems), or they are aimed at advanced rate control operation [40]. In this section, we develop a simple empirical model aimed at deriving a local estimation of the rate distortion curve in 


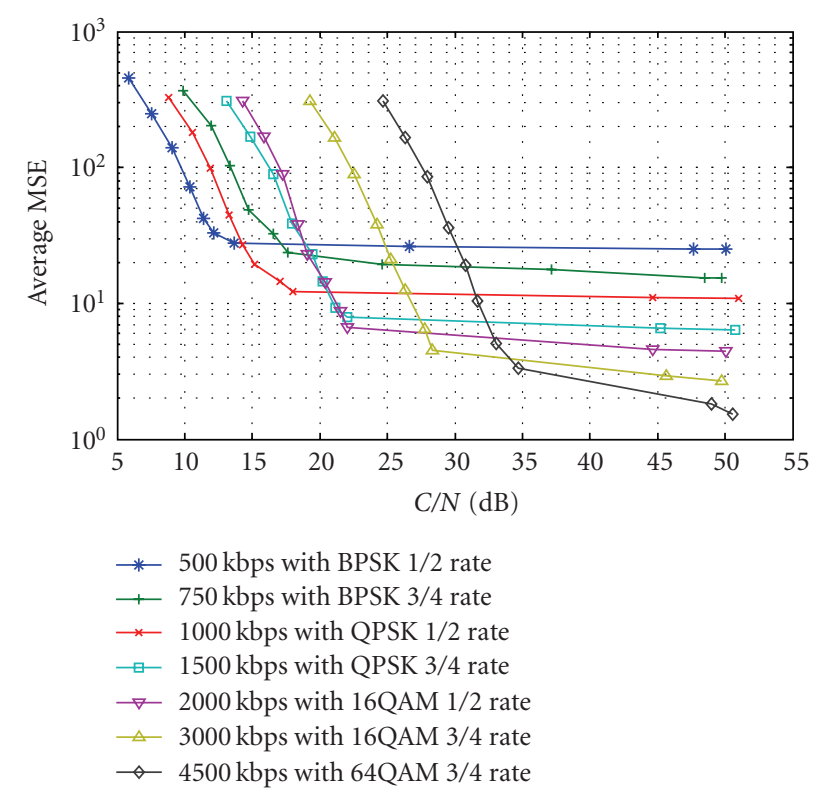

FIGURE 6: Distortion-based link adaptation, foreman, NAL unit max size: 750 bytes.

order to approximate the distortion at lower and higher rates, without relying on multiple encodings, that is, when only one point on the curve is known. The distortion used here is the MSE between the reconstructed and original pixels and is only due to the motion compensation, quantisation and transform operations of the encoder.

We first assume that a GOP has been encoded at the current rate. The actual average coding distortion of the GOP is therefore available, and we estimate the distortion due to coding for the sequence encoded at higher and lower rates. As stated in [41], in H.264, an increase of 6 in the quantisation parameter (QP) approximately halves the bit rate (equivalent to a decrease of 1 in the $\log _{2}$ bit rate). A simple linear relationship between the QP and the $\log _{2}$ of the bit rate can be adopted. As stated in [42], the quantisation design of H.264 allows a local and linear relationship between PSNR and the step-size control parameter QP. This can be expressed mathematically as

$$
\begin{gathered}
\log _{2}(R)=a \times \mathrm{QP}+b, \\
\mathrm{PSNR}=c \times \mathrm{QP}+d,
\end{gathered}
$$

which can be rewritten as

$$
\operatorname{PSNR}=\frac{c}{a} \times \log _{2}(R)+\left(d-\frac{b c}{a}\right) .
$$

This linear relationship between PSNR and the base-two of the logarithm of the bit rate has been verified by plotting the actual PSNR versus $\log _{2}(R)$ for all GOPs in the table (Figure 7(a)) and coastguard (Figure 7(b)) sequences. Similar curves have been obtained with other sequences and we can thus assume that the curves are locally linear, that is, three adjacent points are aligned.

To fully derive the parameters of this linear model, several parallel encodings would be needed, but this is not practical. From the encoding of the current GOP, the current $\mathrm{PSNR}_{c}$ (derived from the averaged MSE), the current rate $R_{c}$ and the current average $\mathrm{QP}_{c}$ are known. Using the fact that an increase of 6 in QP halves the bit rate, we derive $a=-1 / 6$. Moreover, empirical studies for CIF sequences (a similar constant can be obtained for sequences with others resolutions and formats) have shown that trial encodings with a QP of 6 leads to an almost constant luminance PSNR of $55.68 \mathrm{~dB}( \pm 0.3 \mathrm{~dB})$ for akiyo, coastguard, table, and foreman sequences. We can now calculate the four parameters $a, b, c$, and $d$ as

$$
\begin{aligned}
& a=-\frac{1}{6}, \\
& b=\log _{2}\left(R_{c}\right)+\frac{\mathrm{QP}_{c}}{6}, \\
& c=\frac{\mathrm{PSNR}_{c}-55.68}{\mathrm{QP}_{c}-6}, \\
& d=\frac{55.68 \times \mathrm{QP}_{c}-6 \times \mathrm{PSNR}_{c}}{\mathrm{QP}_{c}-6} .
\end{aligned}
$$

To validate this model, video sequences (akiyo, foreman, table, and coastguard) were encoded at the following rates $500 \mathrm{kbps}, 750 \mathrm{kbps}, 1000 \mathrm{kbps}, 1500 \mathrm{kbps}, 2000 \mathrm{kbps}$, $3000 \mathrm{kbps}$, and $4500 \mathrm{kbps}$. Figure 8 (a) shows the estimation of PSNR for the GOP number 10 of the table sequences at 1000 and $2000 \mathrm{kbps}$ (the GOP is encoded at $1500 \mathrm{kbps}$ ). It can be seen that the model follows a similar trend to the actual curve. However, because the reference point $(\mathrm{QP}=6$, PSNR $=55.68 \mathrm{~dB}$ ) may be distant from the current operating point, a mismatch can appear. We have found empirically that weighting the parameter $c$ by a scalar dependent on the average QP improves the accuracy of the model. Figure 8(b) shows similar performance trends with the GOP number 15 of foreman encoded at $3000 \mathrm{kbps}$ when used to estimate the PSNR at 2000 and $4500 \mathrm{kbps}$. Figure 9 shows a comparison between the actual and estimated MSE at the lower and higher rates for all the GOPs of table encoded at $1500 \mathrm{kbps}$ and foreman encoded at $750 \mathrm{kbps}$. Tables 2 and 3 provide the mean and standard deviation of the estimation error calculated over the GOPs, between the actual MSE and the estimated MSEs, for each encoding rate of foreman and table, respectively. It can be seen that the mean error is smaller with the model with linear weighting (and it is below 10\%). Similarly, the standard deviation of the error is smaller when linear weighting is applied and kept in the range from $1 \%$ to $9 \%$. The proposed model employing weighting factors thus offers an acceptable local estimate of encoding distortions for the sequence at lower and higher bit rates.

The procedure to derive the distortion of the current GOP of a sequence as if it was encoded at the lower and higher local (adjacent) rates is summarised as follows.

(i) Derive rate $R_{c}$, average $\mathrm{QP}_{c}$, average $\mathrm{MSE}_{c}$ and $\operatorname{PSNR}_{c}=10 \times \log _{10}\left(255 \times 255 / \mathrm{MSE}_{c}\right)$ from the encoding of the current GOP.

(ii) Derive $a, b, c$, and $d$ using (4). 


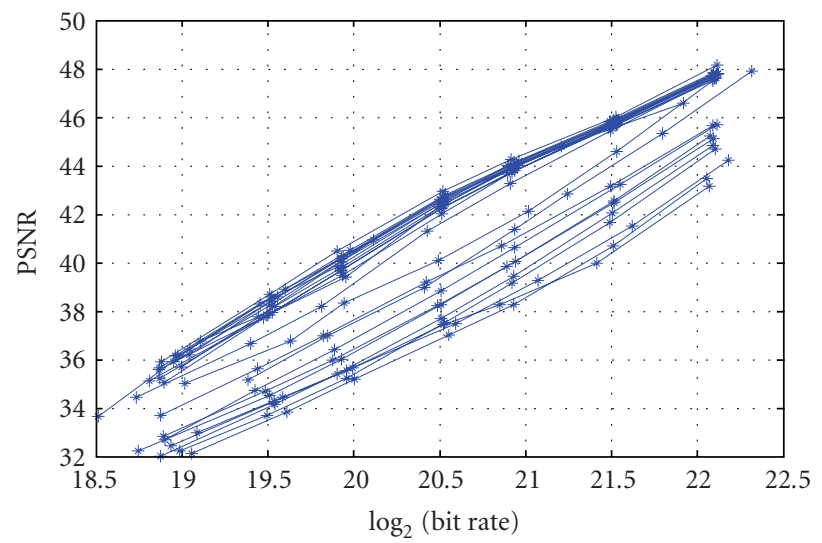

(a) Table

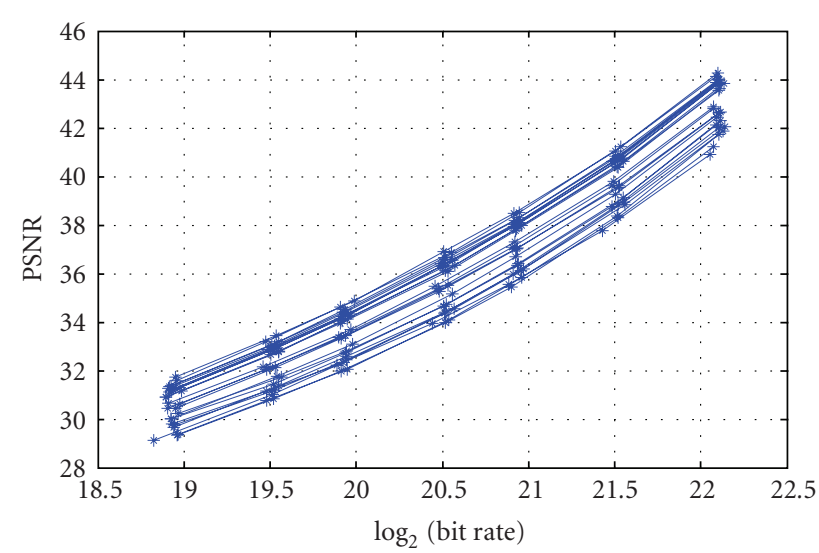

(b) Coastguard

FIGURE 7: PSNR versus $\log _{2}$ (Bit rate) performance for 25 GOPs.

TABLE 2: Mean and standard deviation (calculated over the GOPs) of the estimation error (in percent) between the actual and the estimated MSE, foreman.

\begin{tabular}{|c|c|c|c|c|c|}
\hline \multirow[b]{2}{*}{ Current encoding rate } & \multirow[b]{2}{*}{ Estimation rate } & \multicolumn{2}{|c|}{$\begin{array}{l}\text { Mean of the estimation error } \\
\text { (percentage of difference) }\end{array}$} & \multicolumn{2}{|c|}{$\begin{array}{l}\text { Standard deviation of the estimation error } \\
\text { (percentage of difference) }\end{array}$} \\
\hline & & Linear model & Linear model with weighting & Linear model & Linear model with weighting \\
\hline $500 \mathrm{kbps}$ & $750 \mathrm{kbps}$ & 18.2555 & 7.8208 & 7.0821 & 8.1238 \\
\hline \multirow{2}{*}{$750 \mathrm{kbps}$} & $500 \mathrm{kbps}$ & 25.7355 & 7.4049 & 10.7892 & 6.0400 \\
\hline & $1000 \mathrm{kbps}$ & 16.2241 & 6.3052 & 6.2538 & 3.7887 \\
\hline \multirow{2}{*}{$1000 \mathrm{kbps}$} & $750 \mathrm{kbps}$ & 21.3207 & 7.1663 & 8.8395 & 4.5493 \\
\hline & $1500 \mathrm{kbps}$ & 22.3845 & 6.8882 & 5.2796 & 3.0656 \\
\hline \multirow{2}{*}{$1500 \mathrm{kbps}$} & $1000 \mathrm{kbps}$ & 31.8273 & 8.8351 & 8.2769 & 4.1898 \\
\hline & $2000 \mathrm{kbps}$ & 17.0562 & 5.6035 & 4.2309 & 2.5047 \\
\hline \multirow{2}{*}{$2000 \mathrm{kbps}$} & $1500 \mathrm{kbps}$ & 21.2502 & 6.4256 & 6.0921 & 2.9674 \\
\hline & $3000 \mathrm{kbps}$ & 21.6382 & 5.0351 & 3.5749 & 2.7910 \\
\hline \multirow{2}{*}{$3000 \mathrm{kbps}$} & $2000 \mathrm{kbps}$ & 26.2032 & 4.8640 & 5.1767 & 3.0556 \\
\hline & $4500 \mathrm{kbps}$ & 14.5347 & 4.3805 & 4.0193 & 3.8371 \\
\hline $4500 \mathrm{kbps}$ & $3000 \mathrm{kbps}$ & 16.4630 & 4.0723 & 5.4758 & 3.2906 \\
\hline
\end{tabular}

TABLE 3: Mean and standard deviation (calculated over the GOPs) of the estimation error (in percent) between the actual and the estimated MSE, table.

Mean of the percentage of difference Standard deviation of the percentage of difference Current encoding rate Estimation rate Linear model Linear model with weighting Linear model Linear model with weighting

\begin{tabular}{lccccc}
\hline $500 \mathrm{kbps}$ & $750 \mathrm{kbps}$ & 14.4219 & 12.3402 & 8.2494 & 9.0454 \\
\hline \multirow{2}{*}{$750 \mathrm{kbit} / \mathrm{s}$} & $500 \mathrm{kbps}$ & 19.7089 & 9.4528 & 12.6270 & 5.8535 \\
& $1000 \mathrm{kbps}$ & 11.4824 & 4.9793 & 4.9201 & 3.5082 \\
\hline \multirow{2}{*}{$1000 \mathrm{kbps}$} & $750 \mathrm{kbps}$ & 14.9569 & 4.1785 & 6.2735 & 2.7079 \\
& $1500 \mathrm{kbps}$ & 14.4776 & 9.9738 & 6.5595 & 7.1777 \\
\hline \multirow{2}{*}{$200 \mathrm{kbps}$} & $1000 \mathrm{kbps}$ & 20.4458 & 5.6005 & 5.0650 & 5.1867 \\
& $2000 \mathrm{kbps}$ & 14.6201 & 6.7503 & 9.0542 & 3.3561 \\
\hline \multirow{2}{*}{$3000 \mathrm{kbpss}$} & $1500 \mathrm{kbps}$ & 20.1543 & 10.9368 & 9.5719 & 5.4030 \\
\hline \multirow{2}{*}{$4500 \mathrm{kbps}$} & $3000 \mathrm{kbps}$ & 23.3229 & 15.6379 & 19.3450 & 5.7515 \\
& $2000 \mathrm{kbps}$ & 36.8940 & 14.6120 & 12.8395 & 8.7635 \\
\end{tabular}



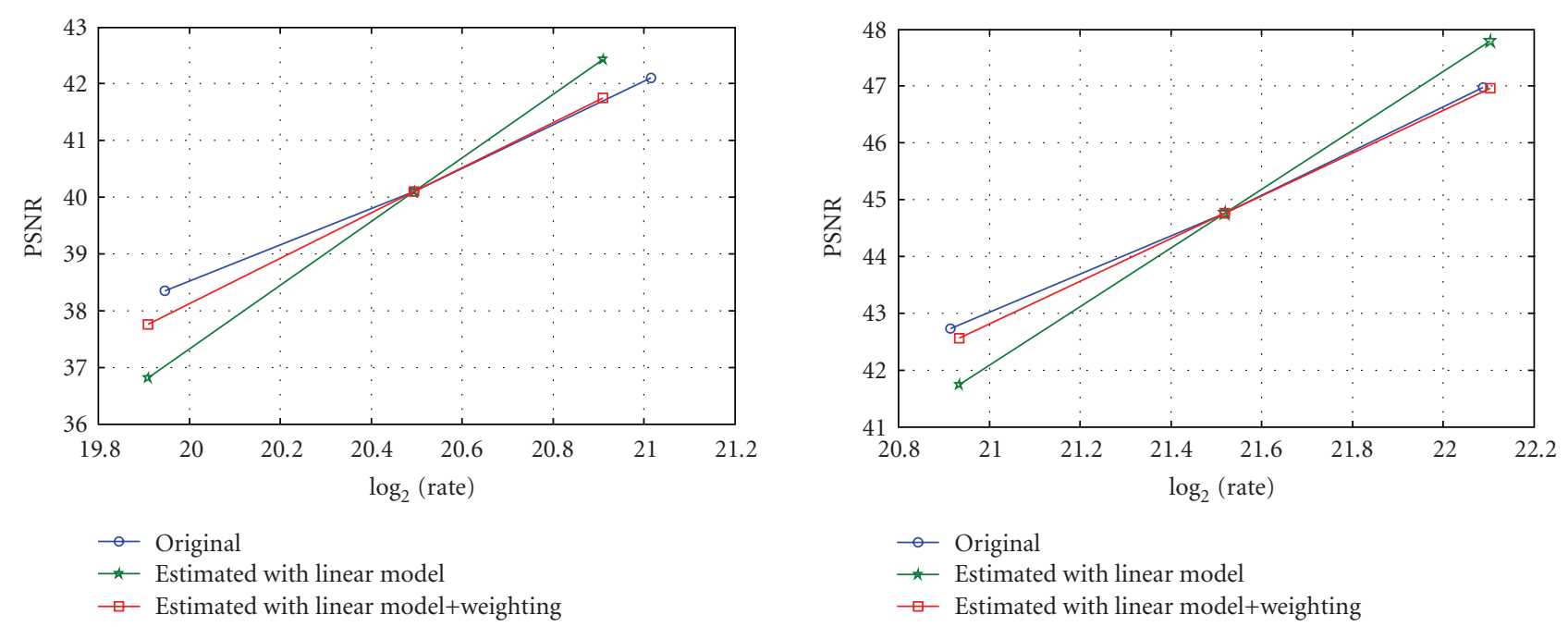

(a) Table encoded at $1500 \mathrm{kbps}$, GOP number $=10$; estimation of the points for encoding at $1000 \mathrm{kbps}$ and $2000 \mathrm{kbps}$

(b) Foreman encoded at $3000 \mathrm{kbps}$, GOP number $=15$; estimation of the points for encoding at $2000 \mathrm{kbps}$ and $4500 \mathrm{kbps}$

Figure 8: Model for the estimation of adjacent encoding points.
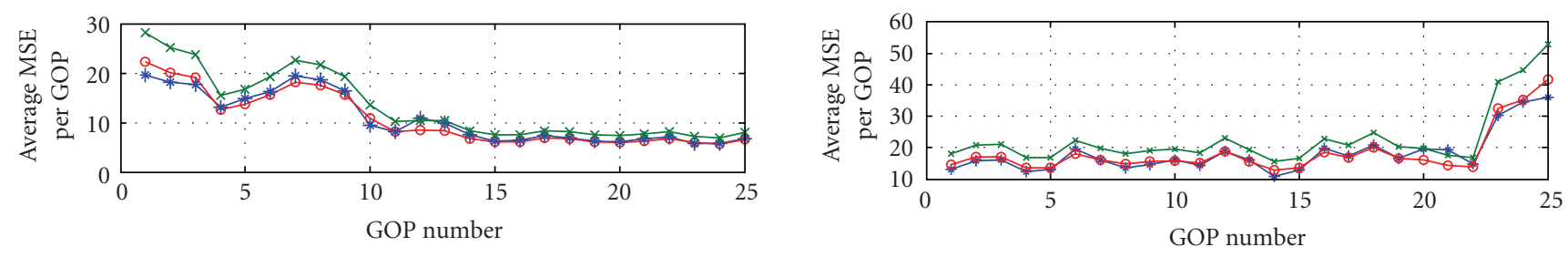

*- Actual $1000 \mathrm{kbps}$

* Estimated $1000 \mathrm{kbps}$ with linear model

$\rightarrow$ Estimated $1000 \mathrm{kbps}$ with linear model+weighting
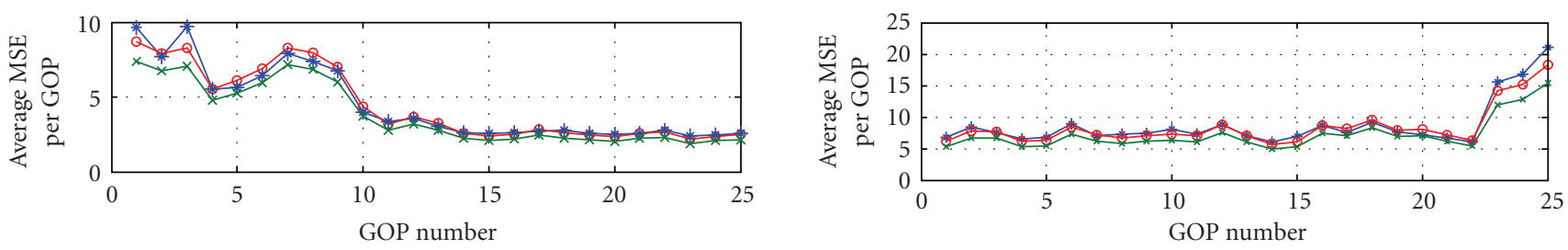

* Actual $2000 \mathrm{kbps}$

$*$ Estimated $2000 \mathrm{kbps}$ with linear model

- Estimated $2000 \mathrm{kbps}$ with linear model+weighting

(a) Table encoded at 1500 kbps: actual and estimated lower rates (1000 kbps, top figure); and actual and estimated higher (2000 kbps, bottom figure) rates

$\rightarrow$ Actual $500 \mathrm{kbps}$

* Estimated $500 \mathrm{kbps}$ with linear model

- Estimated $500 \mathrm{kbps}$ with linear model+weighting

$\rightarrow$ Actual $1000 \mathrm{kbps}$
$\rightarrow$ Estimated $1000 \mathrm{kbps}$ with linear model
$\rightarrow \quad$ Estimated $1000 \mathrm{kbps}$ with linear model+weighting

(b) Foreman encoded at $750 \mathrm{kbps}$ : actual and estimated lower rates ( $500 \mathrm{kbps}$, top figure); and actual and estimated higher rates (1000 kbps, bottom figure)

FIGURE 9: MSE comparison: actual MSE and estimated adjacent MSE.

(iii) Derive $\mathrm{PSNR}_{l}$ and $\mathrm{PSNR}_{h}$ video quality using (2) with the corresponding lower and higher rates $R_{l}$ and $R_{h}$, respectively.

(iv) Compute $\mathrm{MSE}_{l}$ and $\mathrm{MSE}_{h}$ from $\mathrm{PSNR}_{l}$ and $\mathrm{PSNR}_{h}$.

\subsection{End-to-end and transmission distortion model}

To estimate the distortion of the received video, we use the end-to-end distortion model developed in $[38,43]$. We limit the study to only one reference frame; however the model remains valid with a larger number of reference frames. We consider the previous frame copy (PFC) concealment algorithm at the decoder, in which missing pixels due to packet loss during transmission are replaced by the colocated pixels in the previous reconstructed frame. We assume that the probability of a packet loss is $p_{c}$ on the current rate. The current end-to-end distortion for pixel $i$ of frame $n$, noted $\operatorname{Dist}_{e 2 e, c}(n, i)$ accounts for (a) the error propagation from 
frame $n-1$ to frame $n, D_{\mathrm{EP}}(n, i)$; and (b) the PFC error concealment, $D_{\mathrm{EC}}(n, i)$. We therefore have

$$
\operatorname{Dist}_{e 2 e, c}(n, i)=\left(1-p_{c}\right) \times D_{\mathrm{EP}}(n, i)+p_{c} \times D_{\mathrm{EC}}(n, i) .
$$

Readers are referred to $[38,43]$ for full details on how $D_{\mathrm{EP}}(n, i)$ and $D_{\mathrm{EC}}(n, i)$ are derived. Assuming that a pixel $i$ of frame $n$ has been predicted from pixel $j$ in frame $n-1$, $\operatorname{Dist}_{e 2 e, c}(n, i)$ can be expressed as

$$
\begin{aligned}
\operatorname{Dist}_{e 2 e, c}(n, i)= & \left(1-p_{c}\right) \times \operatorname{Dist}_{e 2 e, c}(n-1, j)+p_{c} \\
& \times\left(\operatorname{RMSE}_{c}(n-1, n, i)+\operatorname{Dist}_{e 2 e, c}(n-1, i)\right) .
\end{aligned}
$$

$\operatorname{RMSE}_{c}(n-1, n, i)$ is the MSE between reconstructed frames $n$ and $n-1$ at pixel location $i$ at the current rate. If the pixel $i$ belongs to an intra block, there is no distortion due to error propagation but only due to error concealment; and $\operatorname{Dist}_{e 2 e, c}(n, i)$ is rewritten as

$$
\begin{aligned}
\operatorname{Dist}_{e 2 e, c}(n, i)= & p_{c} \times\left(\operatorname{RMSE}_{c}(n-1, n, i)\right. \\
& \left.+\operatorname{Dist}_{e 2 e, c}(n-1, i)\right) .
\end{aligned}
$$

In order to compute the end-to-end distortion of the sequence transmitted at lower and higher adjacent rates, $\operatorname{Dist}_{e 2 e, l}(n, i)$ and $\operatorname{Dist}_{e 2 e, h}(n, i)$, respectively, with a packet loss of $p_{l}$ and $p_{h}$, respectively, we assume that the motion estimation is similar at all the rates and the difference in quality between the reconstructed sequences is only due to quantisation. Therefore, if pixel $i$ in frame $n$ is predicted from pixel $j$ in frame $n-1$ at the current rate, it will also be predicted from the same pixel $j$ in frame $n-1$ at lower and higher rates. The two distortions at lower and higher rates can then be expressed as

$$
\begin{aligned}
\operatorname{Dist}_{e 2 e, l}(n, i)= & \left(1-p_{l}\right) \times \operatorname{Dist}_{e 2 e, l}(n-1, j)+p_{l} \\
& \times\left(\operatorname{RMSE}_{l}(n-1, n, i)+\operatorname{Dist}_{e 2 e, l}(n-1, i)\right), \\
\operatorname{Dist}_{e 2 e, h}(n, i)= & \left(1-p_{h}\right) \times \operatorname{Dist}_{e 2 e, h}(n-1, j)+p_{h} \\
& \times\left(\operatorname{RMSE}_{h}(n-1, n, i)+\operatorname{Dist}_{e 2 e, h}(n-1, i)\right) .
\end{aligned}
$$

Dist $_{e 2 e, l}$ and Dist $_{e 2 e, h}$ only differ from Dist $_{e 2 e, c}$ by the packet loss and the impact of the concealment algorithm, that is, by $\operatorname{RMSE}_{l}(n-1, n, i)$ and $\operatorname{RMSE}_{h}(n-1, n, i)$. If we consider the lower rate, $\operatorname{RMSE}_{l}(n-1, n, i)$ is given by

$$
\begin{aligned}
\operatorname{RMSE}_{l}(n, n-1, i) \\
=\left[i_{\mathrm{rec}, l}(n)-i_{\mathrm{rec}, l}(n-1)\right]^{2} \\
=\left[i_{\mathrm{rec}, l}(n)-i_{\mathrm{rec}, c}(n)+i_{\mathrm{rec}, c}(n)-i_{\mathrm{rec}, l}(n-1)\right. \\
\left.\quad+i_{\mathrm{rec}, c}(n-1)-i_{\mathrm{rec}, c}(n-1)\right]^{2} \\
=\left[\left(i_{\mathrm{rec}, c}(n)-i_{\mathrm{rec}, c}(n-1)\right)+\left(i_{\mathrm{rec}, l}(n)-i_{\mathrm{rec}, c}(n)\right)\right. \\
\left.\quad-\left(i_{\mathrm{rec}, l}(n-1)-i_{\mathrm{rec}, c}(n-1)\right)\right]^{2},
\end{aligned}
$$

where $i_{\text {rec, },}(n)$ and $i_{\text {rec, } l}(n)$ are the reconstructed pixels at location $i$ from frame $n$ at the current and lower rates, respectively. If we assume that the quality difference between the two rates is evenly spread along the frames of a GOP, the differences $i_{\mathrm{rec}, l}(n)-i_{\mathrm{rec}, c}(n)$ and $i_{\mathrm{rec}, l}(n-1)-i_{\mathrm{rec}, c}(n-1)$ are cancelled. Equation (9) can therefore be rewritten as

$$
\begin{aligned}
\operatorname{RMSE}_{l}(n, n-1, i) & =\left[\left(i_{\mathrm{rec}, c}(n)-i_{\mathrm{rec}, c}(n-1)\right)\right]^{2} \\
& =\operatorname{RMSE}_{c}(n, n-1, i) \\
& =\operatorname{RMSE}_{h}(n, n-1, i) .
\end{aligned}
$$

The error concealment produces a similar contribution to the end-to-end distortion for the current, lower and higher rates. The overall average distortions for each GOP, including the encoding distortion due to quantisation as well as the end-to-end distortion due to error propagation and error concealment, for the lower, current and higher rates, can thus be estimated by

$$
\begin{aligned}
\text { Dist }_{l} & =\text { Dist }_{e 2 e, l}+\mathrm{MSE}_{l}, \\
\text { Dist }_{c} & =\text { Dist }_{e 2 e, c}+\mathrm{MSE}_{c}, \\
\text { Dist }_{h} & =\text { Dist }_{e 2 e, h}+\mathrm{MSE}_{h} .
\end{aligned}
$$

The end-to-end distortion model has been fully validated in $[38,43]$. Figure 10 confirms this by plotting a comparison between the estimated received distortions and the actual transmissions. Figure 10(a) shows the actual received distortion along the GOPs of coastguard encoded at $1500 \mathrm{kbps}$, with PER of $1 \%$, against the estimated received distortion of coastguard when encoded at $1500 \mathrm{kbps}$ (current rate), as well as with the estimated received distortion of the higher rate when encoded at $1000 \mathrm{kbps}$ (from the lower rate) and of the lower rate when encoded at $2000 \mathrm{kbps}$ (from the higher rate). Similar performance is shown in Figure 10(b) for table encoded at $3000 \mathrm{kbps}$ with a PER of $0.1 \%$. Figure 11 shows the estimated distortions on the current, lower and higher rates compared to the actually received distortions for a $\mathrm{C} / \mathrm{N}$ of 23 and $22 \mathrm{~dB}$ for coastguard with the current mode being 5 and 4, respectively. From these figures, it can be seen that the local estimates from our proposed model closely follow the actual received distortion. It should be noted here that the derivation of more complex (and hence accurate) models would effectively provide better performance. However, this is not the primary aim of this paper, and we believe that the proposed models are suitable for our needs.

\section{PROPOSAL FOR IMPROVED VIDEO TRANSMISSION}

\subsection{Algorithm}

The proposed link adaptation scheme assumes that the ratios between the bit rates carried on each mode follow the ratios of the link-speeds available at the PHY layer for each mode. Moreover, it requires that the maximum size of the video packet generated at the encoder is not modified, so that a single PER versus $\mathrm{C} / \mathrm{N}$ lookup table can be used, assuming a single channel type. It is aimed at low-latency video transmission, without reliance on ARQ. The proposed 

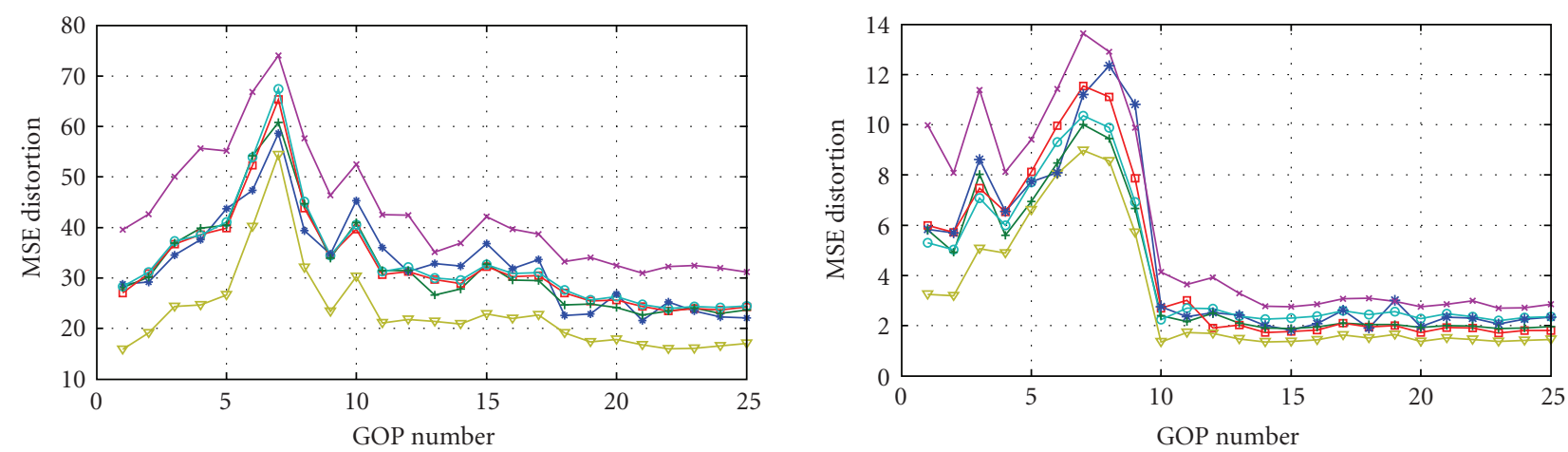

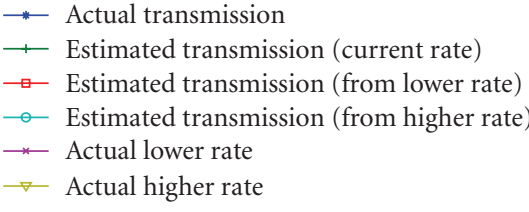

(a) Coastguard encoded at $1500 \mathrm{kbps}, \mathrm{PER}=0.01$

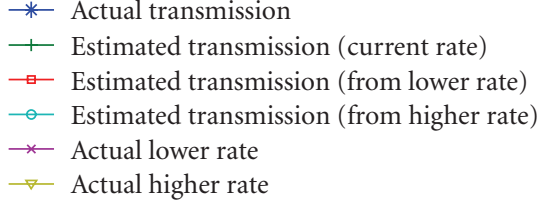

(b) Table encoded at $3000 \mathrm{kbps}, \mathrm{PER}=0.001$

FIgURE 10: Estimated received distortion along the GOPs with fixed PER.

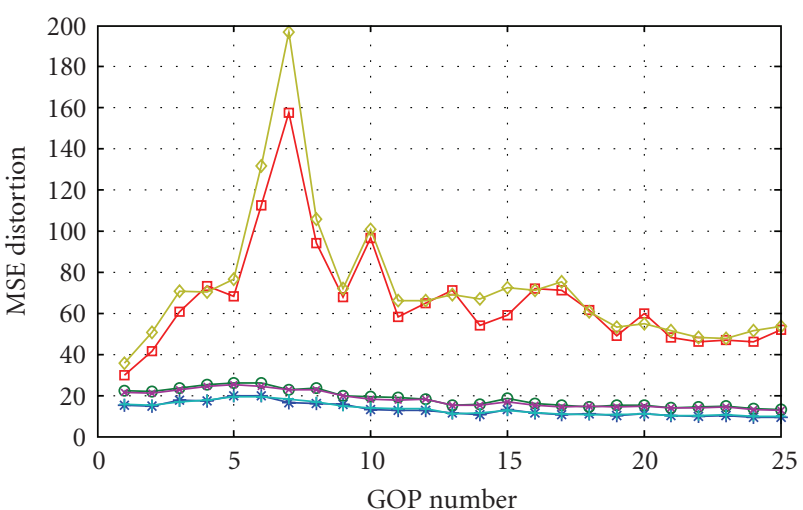

$\rightarrow$ Actual Tx at current rate (mode 5): $2000 \mathrm{kbps}$

- - Actual Tx at lower rate (mode 4): $1500 \mathrm{kbps}$

$\square$ Actual Tx at higher rate (mode 6): $3000 \mathrm{kbps}$

— Estimated Tx at current rate (mode 5): $2000 \mathrm{kbps}$

$\longrightarrow$ Estimated Tx at lower rate (mode 4): $1500 \mathrm{kbps}$

$\checkmark$ Estimated Tx at higher rate (mode 6): $3000 \mathrm{kbps}$

(a) Coastguard, current rate: $2000 \mathrm{kbps}, \mathrm{C} / \mathrm{N}=23 \mathrm{~dB}$

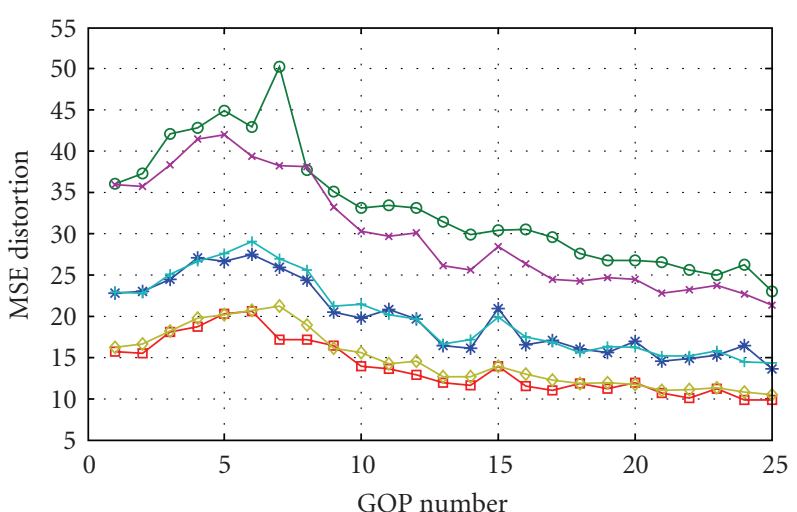

* Actual Tx at current rate (mode 4): $1500 \mathrm{kbs}$

- - Actual Tx at lower rate (mode 3): $1000 \mathrm{kbs}$

$\rightarrow$ Actual Tx at higher rate (mode 5): $2000 \mathrm{kbs}$

$\ldots$ Estimated Tx at current rate (mode 4): $1500 \mathrm{kbs}$

$\rightarrow$ Estimated Tx at lower rate (mode 3): $1000 \mathrm{kbs}$

$\checkmark \quad$ Estimated Tx at higher rate (mode 5): $2000 \mathrm{kbs}$

(b) Coastguard, current rate: $1500 \mathrm{kbps}, \mathrm{C} / \mathrm{N}=22 \mathrm{~dB}$

FIGURE 11: Comparison estimated and actual distortion for different power levels.

algorithm allows dynamic mode switching at each GOP and operates as follows.

(i) Encode the current GOP at the specified bit rate on the specified link-speed.

(ii) Extract the average QP, average MSE, then the average PSNR and average rate $R$ for the GOP.

(iii) Extract the PER from lookup tables using the average received signal strength information (RSSI).

(iv) Derive the estimated distortion at the current, lower and higher modes $\mathrm{MSE}_{c}, \mathrm{MSE}_{l}$, and $\mathrm{MSE}_{h}$ as described in Section 3.1. (v) Compare the distortions:

- if $\mathrm{MSE}_{c}<\mathrm{MSE}_{l}$ and $\mathrm{MSE}_{c}<\mathrm{MSE}_{h}$ : the distortion estimated on the current mode is the lowest; stay in the current mode;

- if $\mathrm{MSE}_{l}<\mathrm{MSE}_{c}$ and $\mathrm{MSE}_{l}<\mathrm{MSE}_{h}$ : the distortion estimated on the lower mode is the lowest; switch to the lower mode, at a lower rate;

- if $\mathrm{MSE}_{h}<\mathrm{MSE}_{c}$ and $\mathrm{MSE}_{h}<\mathrm{MSE}_{l}$ : the distortion estimated on the higher mode is the lowest; switch to the higher mode, at a higher rate. 


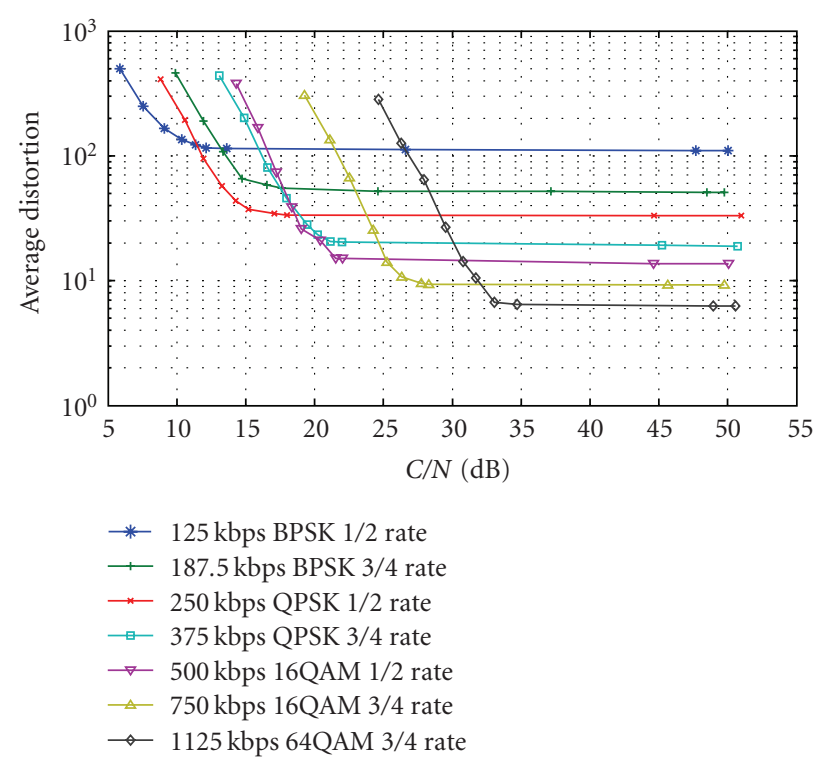

Figure 12: Optimum distortion-based link adaptation, foreman, GOP number 8 , Set (a).

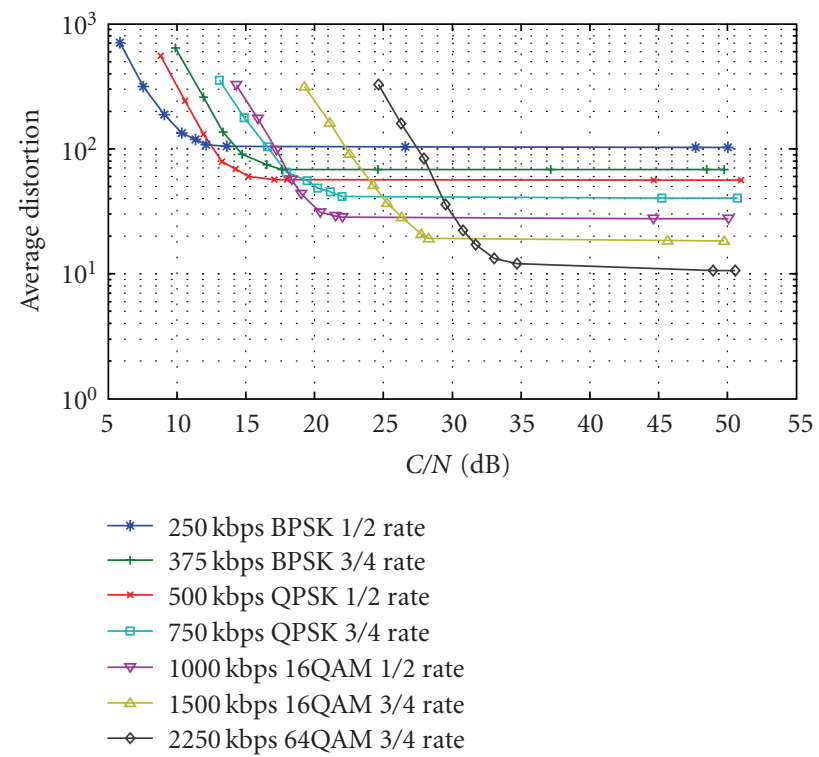

FIGURE 13: Optimum distortion-based link adaptation, coastguard, GOP number 21, Set (b).

(vi) Update the video bit rate at the application layer, update the link-speed at the link layer.

(vii) Proceed to the next GOP and go back to (i).

\subsection{Design and issues}

This algorithm is fully compliant with the IEEE $802.11 \mathrm{a} / \mathrm{b}$ standard and could be implemented in a real system. Moreover, it could coexist with existing algorithms aimed

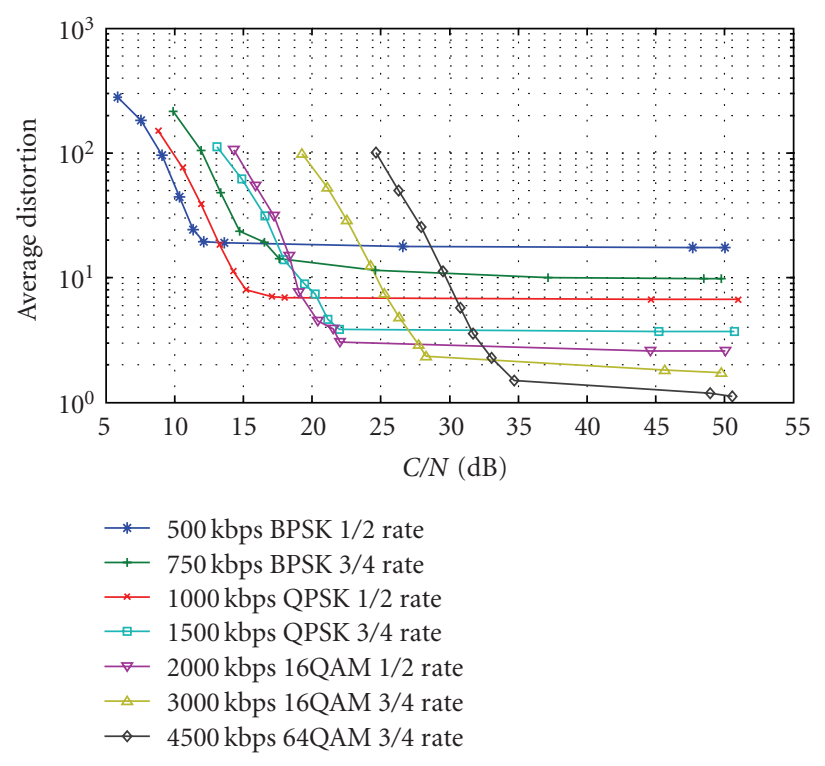

FIGURE 14: Optimum distortion-based link adaptation, table, GOP number 21, Set (c).

at other types of data and could be simply triggered either by a flag or by using access categories, similar to IEEE 802.11e [11] (e.g., packet classifiers are already used to select QoS mechanisms and service flows). The distortion estimation performed at the video encoder does not significantly increase the complexity since it only requires motion compensation, using the already available motion vectors. The change of video bit rate can be achieved either by dynamically changing the target rate in the rate controller (for real-time encoding), or by using a transcoder (for preencoded sequences). Alternatively, a scalable encoder can be employed, dynamically selecting the parts of the bitstream to transmit in order to adjust the bit rate to the bandwidth fluctuations resulting from changes in the link-speed.

The main design issue would be the communication between the application and the link layer. Prior to estimating the distortions, the application layer requires knowledge of the channel conditions from the link layer. Once the switching decision is made, the application layer needs to notify the link layer to update the link-speed accordingly. This exchange of information may be done with a cross-layer communication bus. It should be noted that the frequency of the switching decision can be extended to several GOPs if needed.

With this algorithm, the transmission mode and video bit rate of the current GOP are determined using the channel and video statistics of the previous GOP. A GOP size of 12 frames at 30 frames per second corresponds to a 400millisecond delay. Unless the sequence contains extremely high motion, or scene changes, the motion activity and the sequence content should not be affected by this delay. Moreover, it is reasonable to assume that the overall channel conditions are stable over 400 milliseconds. This value also provides good reactivity to channel changes. However, the 

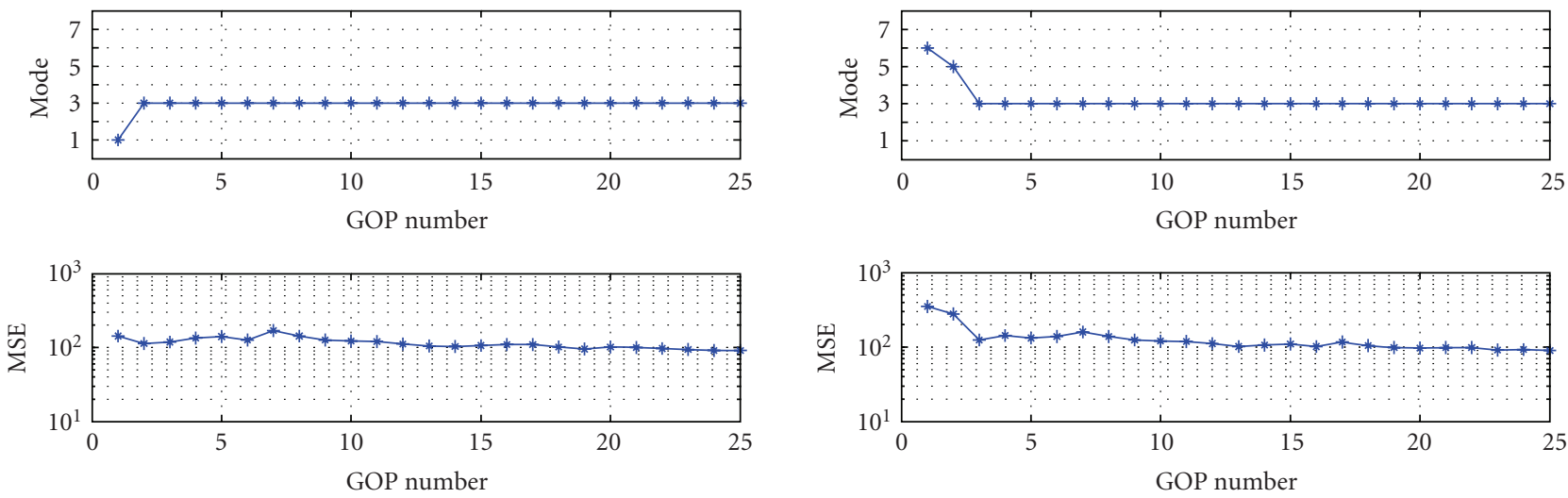

(a) Initial mode $=1($ BPSK $1 / 2$ rate $-125 \mathrm{kbps})$

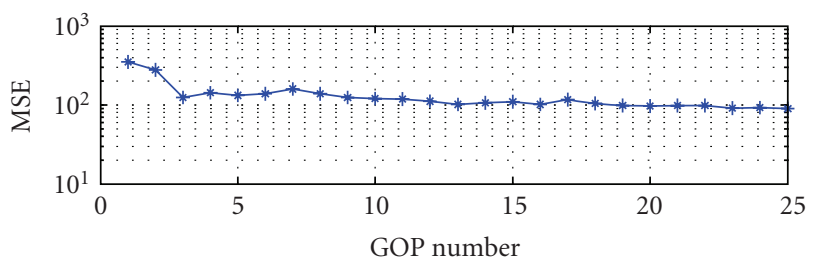

(b) Initial mode $=6(16 \mathrm{QAM} 3 / 4$ rate $-750 \mathrm{kbps})$

Figure 15: Mode and estimated distortion for coastguard encoded with $\mathrm{C} / \mathrm{N}=15 \mathrm{~dB}$, Set (a).
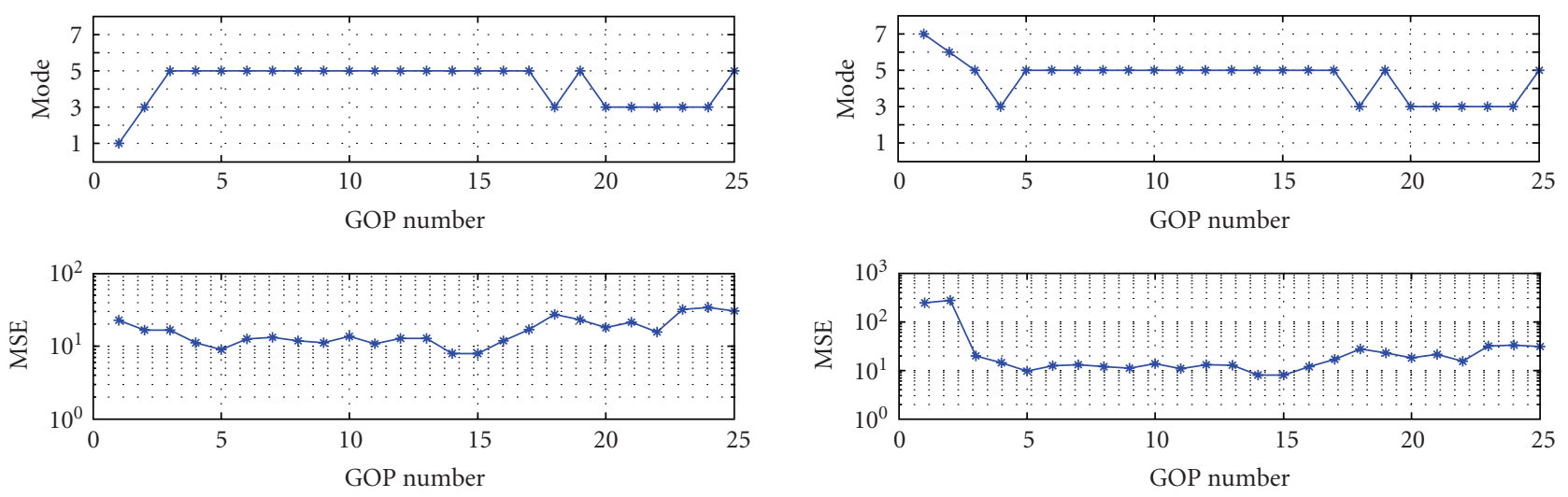

(a) Initial mode $=1($ BPSK $1 / 2$ rate $-250 \mathrm{kbps})$

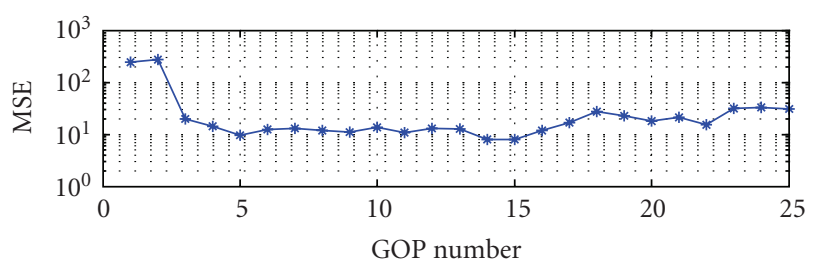

(b) Initial mode $=7$ (64 QAM 3/4 rate $-2250 \mathrm{kbps})$

FIgURE 16: Mode and estimated distortion for foreman encoded with $\mathrm{C} / \mathrm{N}=20 \mathrm{~dB}$, Set (b).

estimated distortions are statistical and might therefore differ from the results of a single transmission.

\section{RESULTS}

\subsection{Simulation conditions}

A compliant 802.11a/g PHY-layer simulator developed at University of Bristol, meeting the conformance requirements specified in Annex A of $[12,13]$, has been used to recreate accurate bit and packet error performance $[22,24]$. The simulator supports all the standardised operating modes and variable PHY-layer packet lengths. Moreover, it implements all the components of the PHY layer with all parameters configured in alignment to the standard and is capable of producing error performance at any $\mathrm{C} / \mathrm{N}$ level. The channel model conforms to the ETSI-BRAN channel A specifications (non line-of-sight office environment), with an rms delay spread of 50 nanoseconds. Using our simulator, an accurate derivation of the PER performance curves and lookup tables, for a PHY packet length of 825 bytes, were produced (in order to fully analyse the proposed mechanism without the influence of others schemes, algorithms which optimally choose packet lengths were not considered, and a simple packetisation with fixed packet length is used in this paper). We assume that packet losses due to collisions are negligible compared to losses due to channel errors.

Four video sequences (akiyo, foreman, table, and coastguard) at CIF resolution are encoded at 30 frames per second (fps) with our modified version of the H.264 reference software [44] (JM version 12.4). Three sets of video rates were considered: (a) from 125 to $1125 \mathrm{kbps}$, (b) from 250 to $2250 \mathrm{kbps}$, and (c) from 500 to $4500 \mathrm{kbps}$. The results presented here are however representative of lower (QCIF, subQCIF) and higher (4 CIF, SD) resolutions as well as lower and higher bit rates; and are used to illustrate the need for cross-layer optimisation and demonstrate the benefits of deploying the proposed system. The RTP format and a fixed maximum NAL unit size of 750 bytes (the 75 remaining bytes account for the RTP/UDP/IP/MAC headers) are chosen. Generated slices are encapsulated into UDP/IP packets. A GOP size of 12, FMO type 2 (dispersed) and one reference frame were used. At the decoder, lost macroblocks (MBs) are simply replaced by the collocated MBs in the previous frame (PFC concealment). 

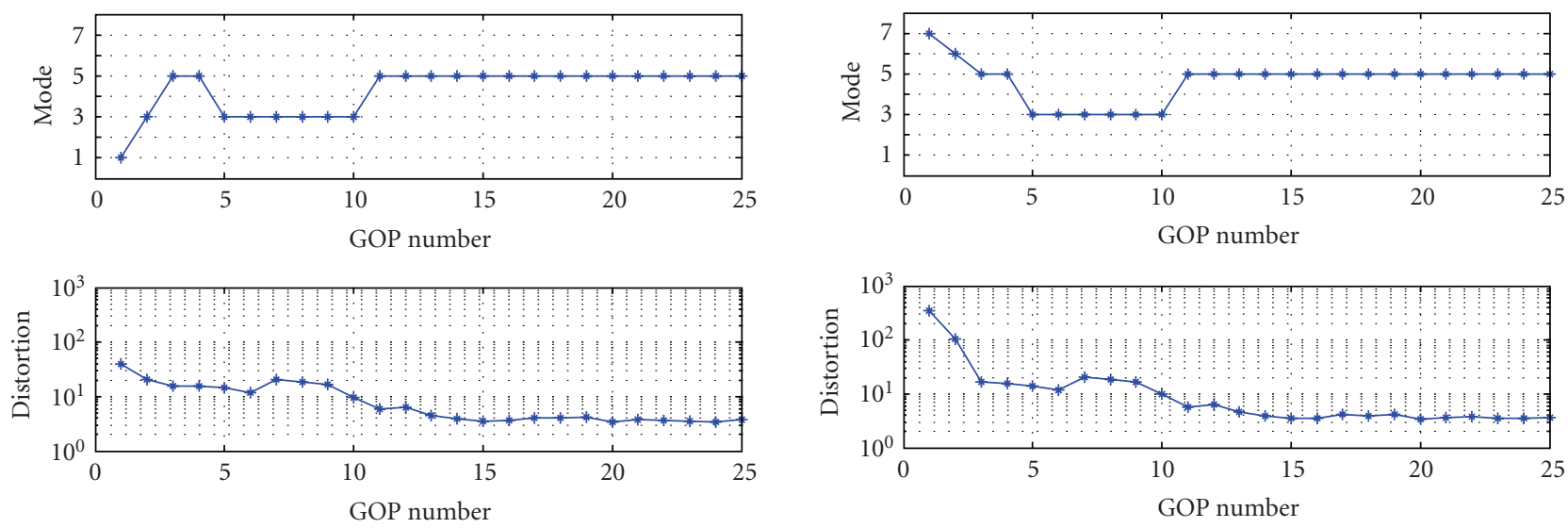

(a) Initial mode $=1($ BPSK $1 / 2$ rate $-500 \mathrm{kbps})$

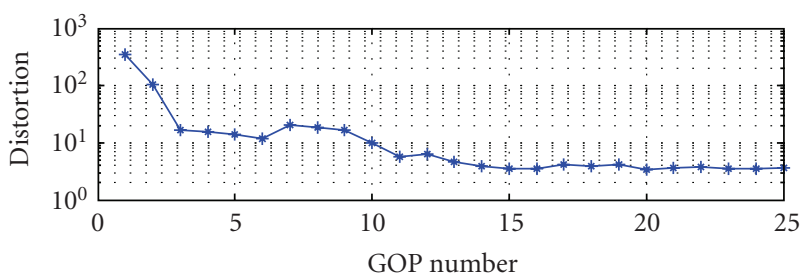

(b) Initial mode $=7(64 \mathrm{QAM} 3 / 4$ rate $-4500 \mathrm{kbps})$

FIgURE 17: Mode and estimated distortion for table encoded with $\mathrm{C} / \mathrm{N}=21 \mathrm{~dB}, \operatorname{Set}(\mathrm{c})$.

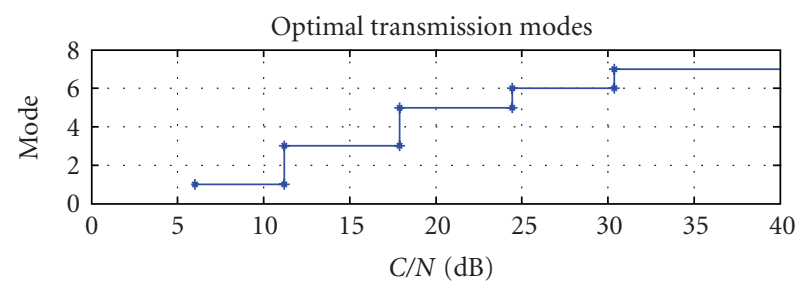

(a) Optimal transmission modes

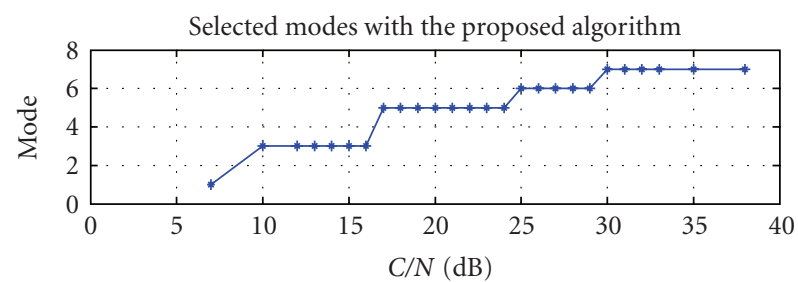

(b) Selected modes with the proposed algorithm

FIGURE 18: Mode selection comparison, table, GOP number 15, initial mode $=3$, Set $(\mathrm{a})$.

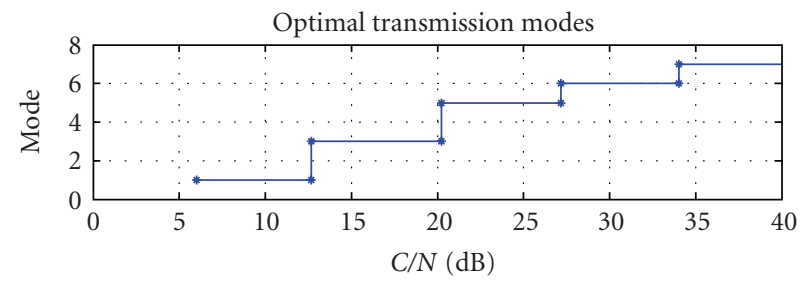

(a) Optimal transmission modes

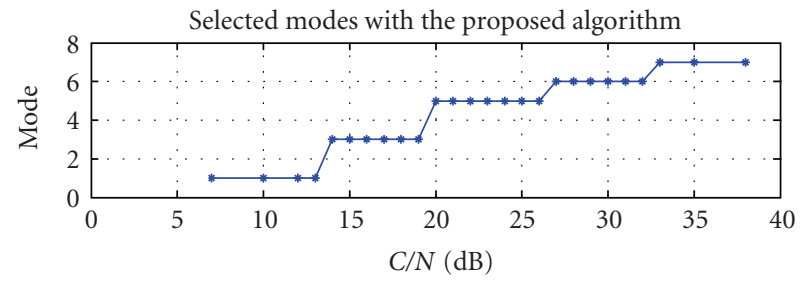

(b) Selected modes with the proposed algorithm

FIgURE 19: Mode selection comparison, foreman, GOP number 11, initial mode $=3$, Set (b).

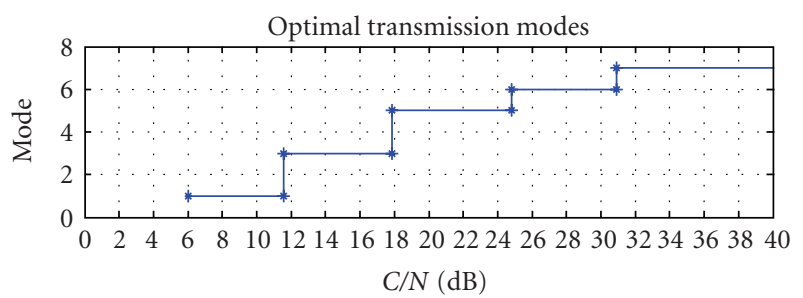

(a) Optimal transmission modes

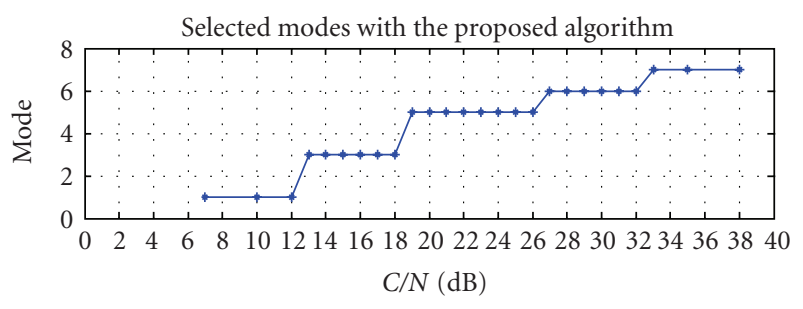

(b) Selected modes with the proposed algorithm

FIGURE 20: Mode selection comparison, akiyo, GOP number 9, initial mode $=6$, Set $(c)$.

\subsection{Optimum link adaptation}

The sequences were encoded at fixed rates, using the three sets: Set (a) 125, 187.5, 250, 375, 500, 750, and $1125 \mathrm{kbps}$; Set (b) 250, 375, 500, 750, 1000, 1500, and $2250 \mathrm{kbps}$; and Set (c) 500, 750, 1000, 1500, 2000, 3000, and $4500 \mathrm{kbps}$ (each of these is transmitted on each of the IEEE 802.11 WLAN modes) and transmitted off-line 50 times (for statistical purposes) over the IEEE 802.11 PHY layer for a wide range of fixed $\mathrm{C} / \mathrm{N}$ power levels. For each sequence, for each GOP, and for each $\mathrm{C} / \mathrm{N}$, the average received distortion (MSE) is calculated and averaged over the 50 runs. This allows us to generate distortion performance curves which will constitute optimum link adaptation, where for each $\mathrm{C} / \mathrm{N}$ the chosen operating mode is the mode with the lowest distortion. Figures 12, 13, and 14 show samples of the optimum link adaptation for GOP number 8 of foreman with Set (a), GOP 
number 15 of coastguard with Set (b), and for GOP number 21 for table with Set (c), respectively.

By examining the PER curves in Figure 1, it can be seen that mode 2 (BPSK $3 / 4$ rate) has worse performance than mode 3 (QPSK 1/2 rate), and that mode 4 (QPSK 3/4 rate) has a similar performance to mode 5 (16 QAM 1/2 rate). Moreover, both offer lower link-speeds (see Table 1). This explains why, using Figure 2 characterising the throughput under various conditions, modes 2 and 4 are never be used. This is also confirmed when examining the optimum link adaptation curves in Figures 12, 13, and 14, where modes 2 and 4 are similarly never used. As a consequence, BPSK 3/4 rate and QPSK 3/4 rate are no longer considered in the remainder of this paper.

\subsection{Behaviour of the proposed system}

The sequences from Section 5.2 have been encoded with our encoder and the proposed cross-layer link adaptation mechanism. This allows the encoder to have knowledge of the $\mathrm{C} / \mathrm{N}$, which is in turn used to estimate the PER of the current mode and also of the adjacent modes. GOP distortion estimates were computed and the target bit rate and operating mode are updated as detailed in Section 4. It should be noted that, for a fixed $\mathrm{C} / \mathrm{N}$, the system behaviour over early GOPs will depend on the initial target and operating mode. This is illustrated in Figures 15, 16, and 17. Figure 15 compares the mode and distortion variations for coastguard encoded with a $\mathrm{C} / \mathrm{N}$ of $15 \mathrm{~dB}$ with two different initial modes, with bit rates from Set (a). With mode 1 as the initial mode, the system upscales rapidly because of the favourable conditions, and then remains steady in mode 3. Whereas starting from mode 6 , the system faces poor channel conditions, and needs to downscale to mode 5 and then to mode 3, where it remains. Similar conclusions can be drawn from Figure 16 with coastguard encoded with a C/N of $20 \mathrm{~dB}$ with rates in Set (b) and from Figure 17 with table encoded with a $\mathrm{C} / \mathrm{N}$ of $21 \mathrm{~dB}$ with rates in Set (c). We also note that the selected mode adapts to channel conditions, but also to video content. For example, in Figure 17, for GOP number 3 to 11 (i.e., from frames 36 to 132) the camera zooms out in the table sequence. This part of the sequence is therefore less resilient to errors and the system automatically switches from mode 5 to 3 ; the sequence remains steady after GOP 11 , where the system upscales to mode 5 .

\subsection{Comparison with optimal link adaptation}

This section compares the optimum modes with the modes selected by our algorithm, as well as the estimated and received distortions with the optimum ones. Figures 18, 19, and 20 compare the selected modes obtained for various $\mathrm{C} / \mathrm{N}$ levels for GOP number 15 of table (Set a), GOP number 11 of foreman (Set b), and GOP number 9 of akiyo (Set c), respectively. It can be seen that the proposed mechanism offers very similar switching points compared to the optimum case. Similar mode switching curves were obtained with other GOP numbers at other $\mathrm{C} / \mathrm{N}$ levels for the three rate sets.

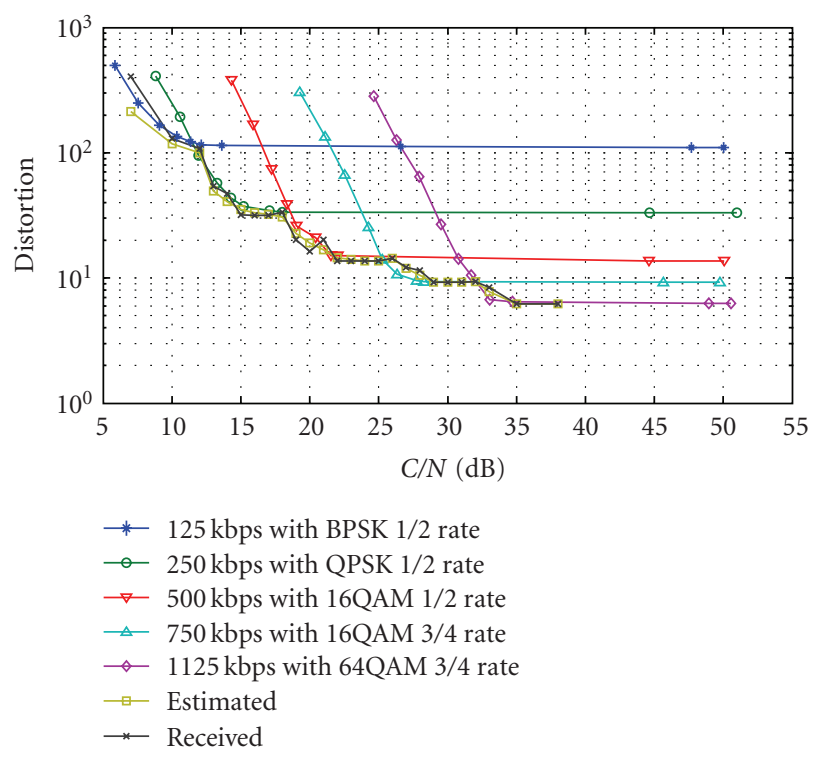

FIGURE 21: Distortion comparison, foreman, GOP number 8, initial mode $=3$, Set $($ a).

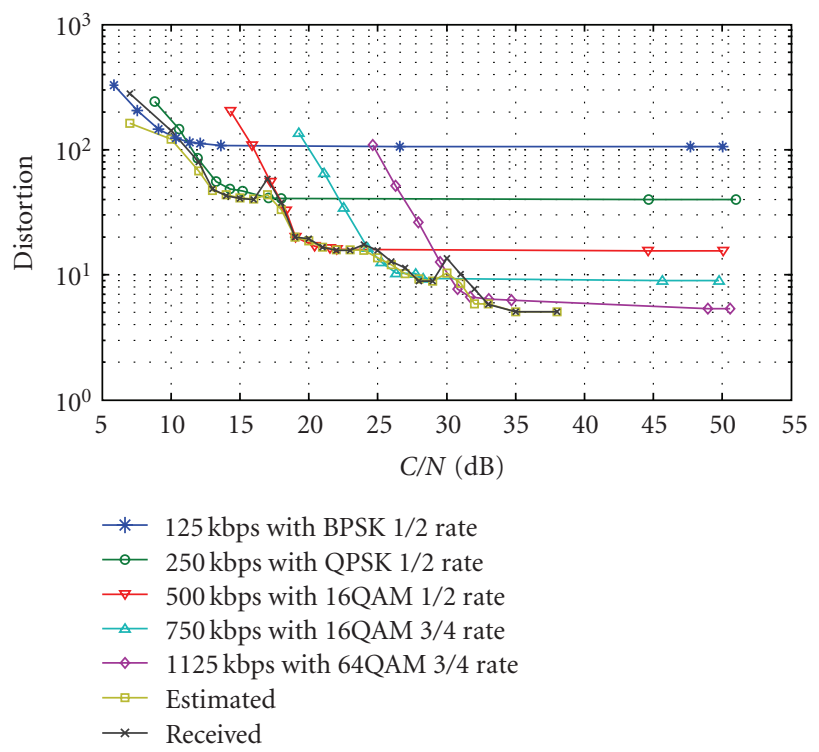

FIGURE 22: Distortion comparison, table, GOP number 15, initial mode $=3$, Set $(\mathrm{a})$.

The simulated curves were obtained by averaging over 50 runs for each video sequence encoded and for each $\mathrm{C} / \mathrm{N}$ level. Figures 21, 22, 23, and 24 compare the optimum link adaptation distortion curves, with the estimated distortion from our system and with the simulated and received distortions, for rates from Sets (a), (b), and (c). First, it can be seen that the estimated and actual distortion levels are very similar, confirming the validity of the proposed model. Moreover, these curves smoothly follow the optimum case. Then, for a given $\mathrm{C} / \mathrm{N}$ power level, the proposed system achieves the lowest video distortion, by adaptively choosing 


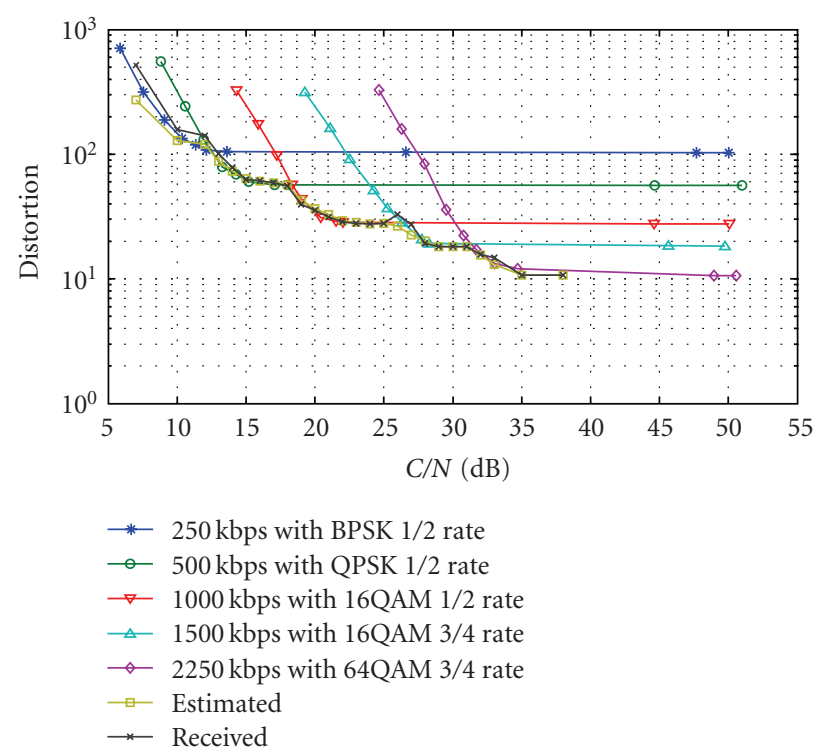

FIGURE 23: Distortion comparison, coastguard, GOP number 15, initial mode $=3$, Set $(b)$.

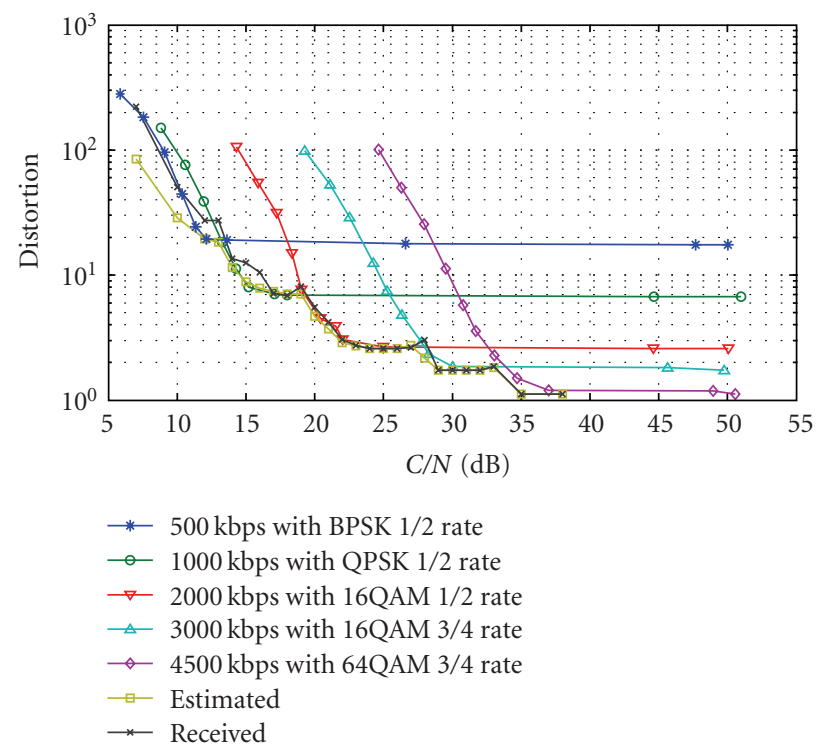

FIgURE 24: Distortion comparison, table, GOP number 21, initial mode $=3$, Set $(\mathrm{c})$.

for each GOP the operating mode which minimises the overall distortion.

\section{CONCLUSIONS}

In this paper, we have presented a novel link adaptation algorithm designed for low-latency video transmission over IEEE 802.11a/g without strong reliance on ARQ. Existing algorithms for link adaptation make extensive use of the retransmission mechanism at the MAC layer in order to improve the error-free data throughput without taking into account the bounded delay requirements of real-time video applications. Moreover, they do not incorporate the spe- cific characteristics of video streams. Completely error-free communication is not essential if robust video compression techniques are used, and it is possible to obtain improved decoded video quality with a stream at a higher bit rate, using a higher link-speed, but with some degree of error rather than an error-free video stream at lower rate, using a lower link-speed. Based on these observations, a link adaptation mechanism minimising the overall transmission video distortion has been presented for low-latency video transmission.

Models were used to estimate the local rate distortion performance at the video encoder and to estimate the endto-end transmission distortion. These models were validated and shown to provide a reasonably accurate estimate of the video distortion. With the assumption that each operating mode carries a different bit rate, the proposed link adaptation uses the estimated overall distortion on the current operating mode, as well as on the lower and higher adjacent modes. For each GOP, the proposed algorithm effectively selects the mode that provides the lowest distortion. A crosslayer exchange of information is needed between the video encoder (at the application) and the link adapter (at the MAC layer).

The proposed system is extendable to other multirate systems such as WiMax and 3GPP LTE, which also support several link-speeds with different modulation and coding rates, each with different reliability levels. Future work will focus on the derivation of more sophisticated rate distortion source models and will also compare the proposed algorithm with known link adaptation techniques such as ARF, for a given received signal level trace. Validations of our approach will be performed using a real-time experimental platform.

\section{ACKNOWLEDGMENTS}

This work was partially funded by the UK TSB project VISUALISE and also by the EU FP6 project ASTRALS.

\section{REFERENCES}

[1] B. Girod, M. Kalman, Y. J. Liang, and R. Zhang, "Advances in channel-adaptive video streaming," Wireless Communications and Mobile Computing, vol. 2, no. 6, pp. 573-584, 2002.

[2] M. van der Schaar, S. Krishnamachari, S. Choi, and X. Xu, "Adaptive cross-layer protection strategies for robust scalable video transmission over 802.11 WLANs," IEEE Journal on Selected Areas in Communications, vol. 21, no. 10, pp. 17521763, 2003.

[3] M. van der Schaar and D. S. Turaga, "Cross-layer packetization and retransmission strategies for delay-sensitive wireless multimedia transmission," IEEE Transactions on Multimedia, vol. 9, no. 1, pp. 185-197, 2007.

[4] M. van der Schaar and S. N. Shankar, "Cross-layer wireless multimedia transmission: challenges, principles, and new paradigms," IEEE Wireless Communications, vol. 12, no. 4, pp. 50-58, 2005.

[5] "IEEE Standard 802.11b-part 11: wireless LAN medium access control (MAC) and physical layer (PHY) specifications: high-speed physical layer extension in the $2.4 \mathrm{GHz}$ band," 1999. 
[6] E. Setton, T. Yoo, X. Zhu, A. Goldsmith, and B. Girod, "Crosslayer design of ad hoc networks for real-time video streaming," IEEE Wireless Communications, vol. 12, no. 4, pp. 59-65, 2005.

[7] I. Haratcherev, J. Taal, K. Langendoen, R. Lagendijk, and H. Sips, "Optimized video streaming over IEEE 802.11 by cross layer signalling," IEEE Communication Magazine, vol. 44, no. 1, pp. 115-121, 2006.

[8] I. Haratcherev, K. Langendoen, R. Lagendijk, and H. Sips, "SNR-based rate control in WaveLAN," in Proceedings of the 10th Annual Conference of the Advanced School for Computing and Imaging (ASCI'04), Delft, The Netherlands, June 2004.

[9] A. Kosentini, M. Naimi, and A. Guéroui, "Towards improvement of the H.264 video transmission over IEEE 802.11e through cross-layer architecture," IEEE Communication Magazine, vol. 44, no. 1, pp. 107-144, 2006.

[10] Joint Video Team of ISO/IEC MPEG ITU-T VCEG, "ITUT H.264-series H: audiovisual and multimedia aystemsadvanced video coding for generic audio visual services".

[11] "IEEE Standard 802.11e; draft supplement to part 11: wireless medium access control (MAC) and physical layer (PHY) specifications: medium access control (MAC) enhancements for quality of services (QoS)," 2002.

[12] "IEEE Standard 802.11g-part 11: wireless LAN medium access control (MAC) and physical layer (PHY) specifications: further high-speed physical layer in the $2.4 \mathrm{GHz}$ band," d1.1 2001.

[13] "IEEE Standard 802.11g; part 11: wireless LAN medium access control (MAC) and physical layer (PHY) specifications: highspeed physical layer in the $5 \mathrm{GHz}$ band," d7.0, 1999.

[14] J. Medbo and P. Schramm, "3ERI085B — channel models for HIPERLAN/2 in different indoor scenarios," ETSI EP BRAN, 1998.

[15] "IEEE Std 802.11; part 11: wireless LAN medium access control (MAC) and physical layer (PHY) specifications," 1999.

[16] I. Haratcherev, K. Langendoen, R. Lagendijk, and H. Sips, "D3.16: application directed automatic 802.11 rate control," Tech. Rep., GigaMobile Project, Technische Universiteit Delft, Delft, The Netherlands, 2002.

[17] P. Ferré, A. Doufexi, J. Chung-How, A. Nix, and D. Bull, "Video quality based link adaptation for low latency video transmission over WLANs," Journal of Zhejiang University: Science A, vol. 7, no. 5, pp. 847-856, 2006.

[18] H. Zhu, M. Li, I. Chlantac, and B. Prabhakaran, "A survey of quality of service in IEEE 802.11e networks," IEEE Wireless Communications, vol. 11, no. 4, pp. 6-14, 2004.

[19] M. Lacage, H. Manshaei, and T. Turletti, "IEEE 802.11 rate adaptation: a practical approach," Tech. Rep., INRIA, Sophia Antipolis, France, 2004.

[20] A. van der Vegt, "Auto rate fall back algorithm for IEEE 802.11a standard," Tech. Rep., HPC Group, Faculty of Physics and Astronomy, University of Utrecht, Utrecht, The Netherlands, 2002.

[21] M. H. Manshaei, T. Turletti, and M. Krunz, "A mediaoriented transmission mode selection in 802.11 wireless LANs," in Proceedings of the IEEE Wireless Communications and Networking Conference (WCNC '04), vol. 2, pp. 12281233, Atlanta, Ga, USA, March 2004.

[22] A. Doufexi, S. Armour, M. Butler, A. Nix, and D. Bull, "A study of the performance of HIPERLAN/2 and IEEE 802.11a physical layers," in Proceedings of the 53rd Vehicular Technology Conference (VTC '01), vol. 1, pp. 668-672, Rhodes, Greece, May 2001.
[23] Z. Lin, G. Malmgren, and J. Torsner, "System performance analysis of link adaptation in HiperLAN type 2," in Proceedings of the 52nd Vehicular Technology Conference (VTC '00), vol. 4, pp. 1719-1725, Boston, Mass, USA, September 2000.

[24] A. Doufexi, S. Armour, M. Butler, et al., "A comparison of the HIPERLAN/2 and IEEE 802.11a wireless LAN standards," IEEE Communications Magazine, vol. 40, no. 5, pp. 172-180, 2002.

[25] D. Qiao, S. Choi, and K. G. Shin, "Goodput analysis and link adaptation for IEEE 802.11 a wireless LANs," IEEE Transactions on Mobile Computing, vol. 1, no. 4, pp. 278-292, 2002.

[26] S. H. Y. Wong, H. Yang, S. Lu, and V. Bharghavan, "Robust rate adaptation for 802.11 wireless networks," in Proceedings of the 12th Annual International Conference on Mobile Computing and Networking (MOBICOM '06), vol. 2006, pp. 146-157, Los Angeles, Calif, USA, September 2006.

[27] D. Qiao, S. Choi, A. Jain, and K. G. Shin, "MiSer: an optimal low energy transmission strategy for IEEE $802.11 \mathrm{a} / \mathrm{h}$," in Proceedings of the 9th Annual International Conference on Mobile Computing and Networking (MOBICOM '03), pp. 161175, San Diego, Calif, USA, September 2003.

[28] G. Holland, N. Vaidya, and P. Bahl, "A rate-adaptive MAC protocol for multi-hop wireless networks," in Proceedings of the 7th Annual International Conference on Mobile Computing and Networking (MOBICOM '01), pp. 236-251, Rome, Italy, July 2001.

[29] I. Haratcherev, K. Langendoen, R. Lagendijk, and H. Sips, "Hybrid rate control for IEEE 802.11," in Proceedings of the 2nd International Workshop on Mobility Management and Wireless Access Protocols (MobiWac '04), pp. 10-18, Philadelphia, Pa, USA, October 2004.

[30] I. Haratcherev, J. Taal, K. Langendoen, R. Lagendijk, and H. Sips, "Automatic IEEE 802.11 rate control for streaming applications," Wireless Communications and Mobile Computing, vol. 5, no. 4, pp. 421-437, 2005.

[31] J. Kim, S. Kim, S. Choi, and D. Qiao, "CARA: collision-aware rate adaptation for IEEE 802.11 WLANs," in Proceedings of the 25th IEEE International Conference on Computer Communications (INFOCOM '06), pp. 1-11, Barcelona, Spain, April 2006.

[32] C. Hoffmann, H. Manshaei, and T. Turletti, "CLARA: closedloop adaptive rate allocation for IEEE 802.11 wireless LANs," in Proceedings of the International Conference on Wireless Networks, Communications, and Mobile Computing (WIRELESSCOM '05), vol. 1, pp. 668-673, Maui, Hawaii, USA, June 2005.

[33] S. Ci and H. Sharif, "A variable data rate scheme to enhance throughput performance of wireless LANs," in Proceedings of the 3rd International Symposium on Communication Systems, Networks and Digital Signal Processing (CSNDSP '02), Staffordshire, UK, July 2002.

[34] W. H. Yuen, H.-N. Lee, and T. D. Andersen, "A simple and effective cross layer networking system for mobile adhoc networks," in Proceedings of the 13th IEEE International Symposium on Personal, Indoor and Mobile Radio Communications (PIMRC '02), vol. 4, pp. 1952-1956, Lisbon, Portugal, September 2002.

[35] M. Lacage, M. H. Manshaei, and T. Turletti, "IEEE 802.11 rate adaptation: a practical approach," in Proceedings of the 7th Symposium on Modeling, Analysis and Simulation of Wireless and Mobile Systems (MSWiM '04), pp. 126-134, Venice, Italy, October 2004.

[36] P. Ferré, A. Doufexi, J. Chung-How, A. Nix, and D. Bull, "Link adaptation for video transmission over COFDM based 
WLANs," in Proceedings of the 10th IEEE Symposium on Communications and Vehicular Technology (SCVT'03), Eindhoven, The Netherlands, November 2003.

[37] K. Stuhlmüller, N. Farber, M. Link, and B. Girod, "Analysis of video transmission over lossy channels," IEEE Journal on Selected Areas in Communications, vol. 18, no. 6, pp. 10121032, 2000.

[38] S. Rane and B. Girod, "Analysis of error-resilient video transmission based on systematic source-channel coding," in Proceedings of the 23rd Picture Coding Symposium (PCS '04), pp. 453-458, San Francisco, Calif, USA, December 2004.

[39] S. Rane, P. Baccichet, and B. Girod, "Modeling and optimization of a systematic lossy error protection system based on H.264/AVC redundant slices," in Proceedings of the 25th Picture Coding Symposium (PCS '06), vol. 2006, Beijing, China, April 2006.

[40] D.-K. Kwon, M.-Y. Shen, and C.-C. J. Kuo, "Rate control for H.264 video with enhanced rate and distortion models," IEEE Transactions on Circuits and Systems for Video Technology, vol. 17, no. 5, pp. 517-528, 2007.

[41] T. Wiegand, G. J. Sullivan, G. Bjontegaard, and A. Luthra, "Overview of the H.264/AVC video coding standard," IEEE Transactions on Circuits and Systems for Video Technology, vol. 13, no. 7, pp. 560-576, 2003.

[42] H. S. Malvar, A. Hallapuro, M. Karczewicz, and L. Kerofsky, "Low-complexity transform and quantization in H.264/AVC," IEEE Transactions on Circuits and Systems for Video Technology, vol. 13, no. 7, pp. 598-603, 2003.

[43] P. Ferré, D. Agrafiotis, and D. Bull, "Macroblock selection algorithms for error resilient H.264 video wireless transmission using redundant slices," in Visual Communications and Image Processing, vol. 6822 of Proceedings of SPIE, San Jose, Calif, USA, January 2008.

[44] K. Suhring, H.264/AVC Software Coordination, http://iphome .hhi.de/suehring/tml/. 